\title{
Disciplinary authenticity and personal relevance in school science
}

\author{
Kapon, Shulamit
}

2018-09

Kapon , S , Laherto , A \& Levrini , O 2018 , ' Disciplinary authenticity and personal relevance in school science ' , Science Education , vol. 102 , no. 5 , pp. 1077-1106 . https://doi.org/10.1002/sce.21458

http://hdl.handle.net/10138/302816

https://doi.org/10.1002/sce.21458

acceptedVersion

Downloaded from Helda, University of Helsinki institutional repository.

This is an electronic reprint of the original article.

This reprint may differ from the original in pagination and typographic detail.

Please cite the original version. 


\title{
Author's accepted manuscript Published in Science Education, 2018 (First published: 13 June 2018; DOI: 10.1002/sce.21458)
}

\section{Disciplinary authenticity and personal relevance in school science}

\author{
Shulamit Kapon, Antti Laherto \& Olivia Levrini
}

\begin{abstract}
Pursuing both disciplinary authenticity and personal relevance in the teaching and learning of science in school generates tensions that should be acknowledged and resolved. This paper problematizes and explores the conceptualizations of these tensions by considering personal relevance, disciplinary authenticity, and common school science as three perspectives that entail different educational goals. Based on an analysis of the literature we identify five facets of the tensions: content fidelity, content coverage, language and discursive norms, epistemic structure and standards, and significance. We then explore the manifestations of these facets in two different examples of the instruction and learning of physics at the advanced high school level in Israel and Italy. Our analysis suggests that: (1) The manifestations of these tensions and their resolution are highly contextual. (2) While maintaining personal relevance and disciplinary authenticity requires some negotiation, the main tension that needs to be resolved is between personal relevance and common school science. (3) Disciplinary authenticity, when considered in terms of its full depth and scope, can be equipped to resolve this tension within the discipline. (4) To achieve resolution, teachers' expertise should include not only pedagogical expertise but also a deep and broad disciplinary understanding.
\end{abstract}

Key words: Engagement, Authenticity, Relevance, Physics Education, High school 
Disciplinary authenticity and personal relevance

\section{Introduction}

Since the beginning of the $20^{\text {th }}$ century, science educators have devoted vast efforts to determining which topics should be taught in science classrooms and what activities students should engage in while learning these topics (Meltzer \& Otero, 2015). In a paper published in Science in 1914, Mann argued that engaging students in project-based learning was a way to awaken their "scientific spirit." He described scientific spirit as "an emotional state, an attitude toward life and nature," claiming that "it is because scientists believe in their hearts that the world is a harmonious and well-coordinated organism, and that it is possible for them to find harmony and coordination, if only they work hard enough and honestly enough and patiently enough, that they achieve their truly great results. It is this faith inside them that inspires them to toil on year after year on one problem" (Mann, 1914).

A century later, the desire to "awaken the students" scientific spirit" still implicitly motivates many initiatives that are informed by pedagogical approaches. Related research strands focus on scientific practices (Barab \& Hay, 2001), professional identity (Hunter, Laursen, \& Seymour, 2007), epistemic practices (Sandoval \& Reiser, 2004), epistemic community (Feldman, Divoll, \& Rogan-Klyve, 2013), and epistemic affect (Jaber \& Hammer, 2016). Science educators are still grappling with how to interweave authentic research experiences into high school and undergraduate science classes, although their motivation and focus may differ from those of Mann's. For example, some may target the emotional "awakening of the scientific spirit," less than a better understanding of how science is done (NGSS Lead States, 2013). Some may consider the use of inquiry as a pedagogical approach that promotes the understanding of scientific content (i.e., inquiry-based learning), whereas others may consider it as a goal in itself, and aim to teach students to meaningfully engage in the practice of doing science. Researchers still face many unresolved issues, such as what students learn when engaging in authentic inquiry (Bell, Blair, Crawford, \& Lederman, 2003; Bleicher, 1996; Charney et al., 2007), what the role of teachers should be (Barab \& Hay, 2001), what kind of support teachers need to be able to advise students on authentic research projects, and the implications for teacher education (Feldman et al., 2013).

Authenticity was traditionally considered to be discipline-driven, and to reflect the desire to simulate "real" scientific practices in the classroom, together with the epistemology and reasoning that such practices entail (Chinn \& Malhotra, 2002; Crawford, 2012). Watkins, Coffey, Redish, and Cooke (2012) defined authentic activities in science "as those that use tools - such as concepts, equations, or physical tools - in ways and for purposes that reflect how the disciplines build, organize, and assess knowledge about the world." (p. 2) This definition focuses on what Irzik and Nola (2014) described as the cognitive and epistemic system of science. The features of this system have also been highlighted by other scholars (e.g., Chinn \& Malhotra, 2002; Crawford, 2012). Yet science can be conceptualized as social-institutional system as well (Erduran \& Dagher, 2014; Irzik \& Nola, 2014), and in fact these social-institutional features account for some of the dialectical processes that shape the role of theory, evidence, explanation and models involved in the development of scientific knowledge (Duschl \& Grandy, 2011). Note however that Mann's (1914) goal of "awakening the students' scientific spirit" goes beyond conceptual, epistemological and even the social-institutional aspects of science. It explicitly requires students to learn not only to think and act like a scientist but also to feel like a scientist. Hence in this view epistemic affect is an organic part of disciplinary authenticity (Jaber \& Hammer, 2016).

Our use of the term disciplinary authenticity encompasses the conceptual, epistemological, socialinstitutional, and affective aspects of doing science. We view disciplinary authenticity, even without the educational layer, as a rich, complex, and context dependent construct, with diverse manifestations. Hence, aiming for disciplinary authenticity in education in our view means designing and facilitating learning experiences that immerse students not only in the conceptual and epistemological features of science, but also in its social dialectical practices, as well as its affective features. In our view disciplinary authenticity in school means that learning experiences must be deeply rooted in and reflect the nature of both contemporary scientific endeavor as well as throughout the history of science. The richness and complexity of the scientific endeavor afford a plurality of manifestations in different educational contexts. The specific nature of disciplinary authenticity in different educational contexts can vary greatly, since while rooted in the nature of the scientific endeavor, its manifestation and meaning in educational settings emerges through 
interactions with teachers, students, and educational social infrastructures that are shaped by different cultures, intellectual interests, institutional constraints, and practical needs. For example, disciplinary authenticity may be manifested in some educational contexts by emphasizing the practices of doing science and generating scientific knowledge, while other, more historical-philosophical oriented settings may emphasize critical reflection on the epistemological and historical processes of the development of scientific knowledge. Engaging students and teachers in the dialectical and social practices of doing or reflecting on science in different social and cultural contexts inevitably leads to the emergence of various contextual meanings and manifestations of disciplinary authenticity (Rahm, Miller, Hartley, \& Moore, 2003). In our view, however, disciplinary authenticity is rich enough to accommodate these variants.

Aiming for disciplinary authenticity in science education at school may present complex and multifaceted tensions. For example, students' engagement in legitimate scientific practices does not always entail the development of knowledge that coheres with canonical content (Hammer, 2001). Moreover, what is considered as "legitimate", who makes this decision, for whom, and for which purpose (Rahm et al., 2003) add additional layers of complexity.

The literature suggests that disciplinary authenticity rarely characterizes the practice of learning science in school (Allchin, 2011; Brown, Collins, \& Duguid, 1989; Chinn \& Malhotra, 2002; Erduran \& Dagher, 2014). Rather, science is transformed for the purpose of teaching and learning science in school. This transformation is a complex and delicate process involving many rationales, influences and constraints that are institutional, educational, political, practical and economic rather than scientific. Thus, the process can result in a construct that is detached from the epistemological, social, dialectical and affective nature of an authentic scientific enterprise.

Erduran and Dagher (2014) argued that school science "rarely coordinates the epistemic, cognitive and social dimensions of science so that learners develop a balanced understanding of what is meant by science in a holistic sense" (p. 40). The Inquiry-Based Science Education (IBSE) approach, for example, which mainly focuses on how to nurture the implementation of the cognitive-epistemic features of authentic science in school, has faced many challenges in its implementation. Authentic activities are complex, openended and require prior knowledge and thinking skills. This has made their transformation into instructional activities that are accessible by all students but still retain their essential authentic features a challenge (Edelson, 1998; Lee \& Butler, 2003). Chinn and Malhotra (2002) argued that the complexity, equipment, procedures, theories, expertise, and techniques for data analysis and modelling that characterize authentic inquiry can rarely be reproduced in most school settings.

The distinction between disciplinary authenticity and school science can also be understood in terms of culture. School culture has a profound influence on what is learned in school (Lave, 1997), and due to unbridged cultural differences schoolwork often becomes inauthentic (Brown et al., 1989). Our use of the term common school science refers to science as it is often taught to and experienced by students in school as a result of prevailing cultural and institutional constraints.

Recent discussions on authenticity in science education have argued implicitly, and often explicitly, for student and teacher agency in determining the meaning of authenticity. In these debates, authenticity is seen as emerging from the interaction between canonical science, teachers and students (Rahm et al., 2003) or resulting from positioning learners in collaborative social contexts so they can assume ownership of the scientific questions they explore, at least to some extent (Calabrese-Barton, 2001; Rivera Maulucci, Brown, Grey, \& Sullivan, 2014). Buxton (2006) examined the various ways in which the term authenticity is perceived in science education. He considered the "youth-centered perspective on authenticity" to be the extreme alternative to the canonical science perspective on authenticity. According to the youth-centered perspective, learning is only authentic when it originates from students' desires, interests and needs. However, studies that have adopted this perspective have usually been conducted in informal education settings, such as enrichment programs that target young children from families with multiple socioeconomic related problems (Calabrese-Barton, 1998, 2001). In this type of context, it is easy to accept that students' needs and interests should come before disciplinary demands. Here we problematize this latter claim and examine the extent to which it is indeed "easy to accept" when extrapolating this logic to science education at the undergraduate and advanced high school levels, where accountability to the discipline must 
be adhered to. Our perspective seeks to resolve this conundrum through student engagement that entails both components simultaneously.

This tension is also implicitly present in debates over the relevance of science education. Relevance was traditionally considered to be personally and societally driven and thought to be a central attribute of science education that could be productively applied to daily life decisions and future careers (Hurd, 1998). A recent comprehensive review of the concept of relevance as it is used in the science education research literature (Stuckey, Hofstein, Mamlok-Naaman, \& Eilks, 2013) found the term to be inadequately conceptualized. To clarify its meaning, the authors suggested an organizing scheme that defines relevance along three dimensions - individual, societal and vocational — each of which applies both intrinsically and extrinsically to students' presents and futures. Given the extensive use of the term, it is surprising that relevance to content and the epistemological and cultural nature of science, technology, engineering and mathematics disciplines (STEM) have not been explicitly considered in analyses of the relevance of science education.

Whereas the discussion on relevance has tended to focus on students or on society as legitimate agents in defining what is relevant in science education, the opposite trend characterizes the debate on authenticity. Here the canonical disciplines have been the main determining agents, and only a few progressive perspectives have included students, teachers and society as legitimate agents in defining authentic science learning. When seen this way, the boundaries between the relevance and the authenticity of science education become fuzzy, and the modern meanings of both concepts seem to emerge from interactions among canonical STEM disciplines, students, teachers, and the society in which all these agents operate.

The meaning we ascribe to the term personal relevance is drawn from the literature on relevance as well as the contemporary discussions of authenticity cited above. We also build on our own work on students' sensemaking (Kapon, 2017; Kapon \& diSessa, 2012) and appropriation (Levrini, Fantini, Pecori, Tasquier, \& Levin, 2015; Levrini, Levin, \& Fantini, 2018). For us, personal relevance encompasses students' sense of benefit, value, and meaningfulness, as well as agency as users and generators of what is learned. By meaningfulness we mean that what is learned make sense to students at a personal level and is something they actively strive to achieve. For example, students try to generate, evaluate, and refine explanations to themselves, rather than explaining to the teacher, and are emotionally invested in this process.

The tensions between disciplinary authenticity, personal relevance, and school science appear implicitly and often explicitly in scholarly debates on authenticity and relevance. The goal of this paper is to problematize the conceptualizations of the tensions between disciplinary authenticity, personal relevance and common school science, by examining specific manifestations of these tensions in learning environments that explicitly aim to foster both disciplinary authenticity and personal relevance in school science.

\section{The tension in the literature}

We start by examining the literature that discusses the tensions that arise in STEM learning environments in which teachers aim to promote disciplinary learning while fostering students' sense of authority, agency, and identity with regard to the subjects learned. Our review focuses on studies that provide detailed accounts of teaching and pedagogical design and aims to elicit and highlight the different facets of these tensions, and the contextual nature of their manifestations.

\section{Content fidelity and content coverage}

Ball (1993) discussed the dilemma of respecting children as thinkers vs. teachers' responsibility for particular content. Specifically, Ball reflected on her experience as a third- grade mathematics teacher and recalled an incident in which one child, Sean, suggested that the number 6 could be both even and odd. Ball explained that on the one hand she felt, as teacher, the need to correct Sean. On the other hand, this was a creative idea that reflected deep thought on the part of the student and dismissing it might reflect 
disrespect to the student as a mathematical thinker. Ball resolved this tension (or dilemma in her words) by acknowledging the originality of the idea and using it to teach the students about the nature and role of definitions in mathematics, and how mathematics knowledge evolves. She told the class that Sean had invented another kind of number that can be both even and odd, termed it the "Sean number," and pressed Sean for a more articulated definition of "Sean numbers." In the next lessons, she allowed children who were interested to explore the patterns of "Sean numbers" as they explored odd and even numbers. Note, however, that the tension was still present. Ball was worried that exploring "Sean numbers" might have confused the children (at least potentially), and to make sure this was not the case, she gave them a quiz about odd and even numbers to make sure they fully understood their properties.

Ball's dilemma highlights a particular facet of the tension we wish to explore. We term this facet content fidelity. The perspective of common school science (school mathematics in this case) requires that students learn correct and acceptable scientific ideas as they are expressed in the curriculum. On the other hand, the personal relevance perspective suggests that students should develop their competence and perception of themselves as able individual thinkers who can create knowledge, which cannot be done without making mistakes. Ball resolved this tension by employing the perspective of disciplinary authenticity. She used the incident as leverage to teach and engage the children in a deep epistemological facet of doing mathematics; namely generating and working with definitions. Note that disciplinary authenticity also entails accountability to particular content. However, while from a common school science perspective the content is defined by the curriculum, from a disciplinary authenticity perspective the canonical content of science emerges from a complex, fluid, although verified dialogue that results in intersubjective conventions and shared knowledge. By telling the children that Sean had invented a new number Ball explicitly introduced the notion of creativity in authentic science. Creative and inventive thought constitutes the seed of any canonical idea, and it precedes the processes of argumentation, testing, evaluating and peer-reviewing in science. Ball in fact engaged the children in an authentic examination of Sean's invention. Hence content and practice did not present any tension from a disciplinary authenticity perspective per se, but they did present a tension from a common school science perspective, since "Sean numbers" were not part of the curriculum.

Hammer (1997) described a somewhat similar tension in $11^{\text {th }}$ and $12^{\text {th }}$ grade physics classes he taught. The activities involved worksheets on electrostatics developed by Robert Morse, which engaged the students in a guided inquiry about basic phenomenological properties of conducting and isolating materials, and the construction of the canonical model for these phenomena. One of the groups came to the conclusion that electrical charges can move from one object to another if the touching objects are made from the same material. From a physicist's perspective this conclusion disregards the canonical distinction between conductors and insulators. The group invented an experiment to test this idea, and according to their findings, the idea appeared valid (i.e., an electrical charge can move from foam to foam, in complete contrast to the scientifically accepted model, and their everyday experience). Hammer reports that he was "torn" about how to assess the students' work and how to proceed.

And so, in this moment, I am torn. I could help them design and perform their experiment "more carefully," so that it shows the correct answer. Perhaps they will come to accept that aluminum conducts and foam does not, but perhaps they will have less of a sense of their own access to physical phenomena, independent of my authority. (Hammer, 1997, p. 489)

Unlike Ball's "Sean numbers" that involved an imaginative definition, from a physicist's perspective the work of Hammer's students reflected a clear-cut mistake in terms of theory and experimental procedures, since electrical charges do not move between two insulators that merely come in contact. Hammer noticed the problem in the experimental design ("Watching their experiment, I saw...", p. 500), and decided to intervene and draw the students' attention to it ("Well what if I put the cup over here ...", p.500), and thus help them reconstruct the experiment so that it would give them the appropriate result. But he remained torn.

I helped them reconstruct the experiment to give the appropriate result, but I was uncomfortable, largely because of my sense of the different needs of the different students. For Jean in particular, I worried my intervention would corroborate what she suspected all 
along - that, in the end, this was about "getting the right answer," and all of this exploration was a kind of sham. (Hammer, 1997, p. 500)

At first glance the tension described above seems to be an issue of content fidelity. The students (i.e., Jean) experienced it as a tension between the perspective of common school science, which is expressed by the requirement to get the right answer, and personal relevance, which calls for individual sensemaking (i.e., self-generated explanations that are targeted to oneself rather than the teacher, and aim to make sense of the phenomena in question, see Kapon, 2017). However, from the teacher's point of view, this case is substantially different and more complex than the previous one. On the one hand the conclusion the students reached was an evident contradiction with accepted scientific knowledge. On the other hand, it stemmed from a creative engagement in inquiry, and an experiment that the students invented. The problem was in the detailed experimental design. Hammer suspected that some of his students may have interpreted his intervention as telling them how to perform the measurement to "get the right answer" instead of learning how to conduct and regulate a reliable scientific investigation.

Hammer (1997) described the tension as taking place among his objectives, and considered himself (i.e., the teacher) and his students as the only agents that shaped it. We argue that the background assumptions of common school science played a pivotal role in shaping this tension. We suspect that if Hammer, as the teacher, had not been bound to the institutional constraints of common school science, such as the need to respect a time schedule and cover a particular set of topics in certain period of time, he could have, for example, engaged the students in a reexamination of the assumptions and process of their measurements, and invited them to use authentic scientific practices to problematize their conclusions and regulate, refine and correct their experimental design and the substantiated reasoning. Thus, the tension did not emerge from the content-practice nature of disciplinary authenticity per se, but rather from the tension between common school science and disciplinary authenticity; i.e., having the time to engage in the practice of doing science without the overriding pressure to cover a certain number of topics during the school year.

To maintain disciplinary authenticity and personal relevance, teachers should primarily direct their attention to the substance of students' ideas, acknowledge the disciplinary connections between these ideas, take them up and pursue them. However, for this to really take place, the curriculum cannot be determined entirely in advance, since it is largely discovered and emergent, and thus presents challenges if the teacher has to cover an externally-predetermined specific set of topics within a certain amount of time (Hammer, 1997; Richards \& Robertson, 2016). We term this kind of tension content coverage. Note that maintaining a comparable educational level between schools potentially affords mobility and equal opportunities. Coordinating the needs of individual students, within the needs of the whole class, within the needs of a larger society, manifests the tension related to content coverage between personal relevance and common school science. Its resolution can be very challenging for teachers.

Both Hammer and Ball conducted their studies in traditional classroom settings. Both highlighted the central role of the teacher in resolving the tensions they describe. Specifically, they argued that the teacher is called upon to identify productive students' mistakes and help the students construct scientific understanding through reasoning and elaboration of these ideas. Ball and colleagues (Ball, Sleep, Boerst, \& Bass, 2009) discussed how this ability can be systematically nurtured in preservice teacher education. Hammer and colleagues (Hammer \& Zee, 2006) discussed how in-service teachers can learn to listen and notice the science in their students' thinking.

The manifestation and the resolution of content coverage and content fidelity dimensions are different in different disciplines and educational contexts. The specificity of the dilemmas depends on the discipline taught. For example, experimental procedures are less relevant in mathematics as compared to biology and right-wrong answers are less compelling in the interpretation of a poem. The attributes of the educational context such as students' grades, the level of scientific complexity of the course, the nature of the activity (inquiry, whole class discussion, etc.), the social infrastructure in which the activity is embedded (the particular settings of formal/informal education), and the students' characteristics all influence how tensions related to content fidelity and content coverage are manifested and can be resolved. 


\section{Language and discursive norms}

Arguments in the literature about tensions between disciplinary accountability, personal relevance, and common school science are not only about content. Some of the facets of these tensions have cultural origins. Rosebery, Warren and Conant (1992) observed language-minority middle school students who were studying science. They noted that appropriating scientific discourse is a particularly challenging task for these students since the distance they must travel between discourse worlds is greater. Hence the tension the teachers were required to resolve was between the language and discursive norms of the culture to which the students belonged (personal relevance) and the language and discursive norms of science (Warren, Ballenger, Ogonowski, Rosebery, \& Hudicourt-Barnes, 2001). We term this facet of the tension language and discursive norms. Warren et al. (2001) argued that the first step in resolving this type of tension is to acknowledge students' cultural discursive patterns as legitimate and productive resources on which scientific discursive patterns can be constructed (as opposed to inhibiting discursive patterns that should be replaced). For example:

As we observed how these children approached the problem of designing an experiment in ways that seemed outside the canonical approach-imagining themselves into the ants' world, evaluating claims, constructing rather than identifying variables — we had to confront our own assumptions about the nature of experimental thinking as exclusively a mode of logical inference. As we examined Emilio's work, we were struck by how his practice of imaginatively inhabiting the experimental world — what at another time we might have seen as anthropomorphism — was actually a generative practice for exploring the created world of the experiment to evaluate relevant knowledge claims. This process led Emilio to see in what ways specific material elements (i.e., variables and conditions) of the experimental world were confounded and how to design the world in a way which looked in the end fully canonical. (Warren et al., 2001, p. 547).

In our view when Warren et al. wrote that "we had to confront our own assumptions about the nature of experimental thinking as exclusively a mode of logical inference" the assumptions they were confronting were those of the perspective of common school science, not disciplinary authenticity. Emilio was performing a thought experiment in the context of middle school biology which is an authentic scientific practice. The history of physics is full of famous examples of the use of thought experiments in scientific investigations by prominent scientists such as Galileo, Maxwell and Einstein. Thought experiments are also employed in authentic investigations in biology, although they are not as common, nor as famous, as they are in physics (Buzzoni, 2015; McAllister, 1996). However, thought experiments are scarcely employed in basic scientific textbooks (Gilbert \& Reiner, 2000); hence they are not part of the discursive norms in common school science.

In this sense, the tension related to language and discursive norms that the teacher had to resolve was between Emilio's imagination and the discursive norms perceived as "acceptable" in school science or, in other words, a tension between personal relevance and common school science. As in the case of Hammer and Ball, in principle, this tension could be resolved within the discipline. However, again as in the case of Hammer and Ball, the disciplinary background of the teacher constituted another layer of complexity to this resolution. To be able to notice and capitalize on discursive norms that may not be part of school science but cohere with authentic scientific discourse, teachers need to have a very strong background in the discipline and feel confident with its epistemic practices of modelling, explaining and arguing.

\section{Epistemic structure and standards}

A related but different culturally-based tension is described in Bang's and Medin's (2010) work, which highlights a culturally-based tension related to epistemic structure and standards. Building on a decade of close collaboration with Native American communities, as well as studies that described and 
analyzed ways in which ethnocentrism plays out especially with regard to epistemology, indigenous traditions, and Western-European traditions, Bang and Medin highlighted the differences in "epistemological orientations" between indigenous traditions and science as a product of the WesternEuropean culture. For example, Bang, Medin, \& Washinawatok (2010) discussed how Menominee fishermen tend to organize knowledge and reason along ecological rather than taxonomic lines by focusing on the relations of organisms to one another and their physical surroundings instead of their systematic classification. In contrast, an analysis revealed that most middle school text books employ a taxonomic approach, and hardly ever use system-level analyses or discuss ecosystems as an organizing principle (Bang et al., 2010).

Bang and Medin (2010) suggested that one way to resolve this tension would be by shifting the orientation of science education from aiming to have students adopt specific epistemologies at the expense of others to supporting students' navigation between multiple legitimate epistemologies. In the spirit of Ball (1993), Hammer (1997) and Warren et al. (2001), Bang and Medin suggested that indigenous cultural ways of experience and knowing (i.e., indigenous epistemologies) could become productive resources to study middle school biology. They developed and implemented community-based summer science programs according to these principles.

The curriculum in the program was intentionally collaboratively designed with members of Native American communities. The topics were chosen according to their relevance to the community's heritage and culture ("locally meaningful interventions focused on ecosystems"), and the content was reconstructed in ways that recognize and honor indigenous epistemological practices and orientations as relevant to science and science learning (Bang et al., 2010). "Navigation between cultures" was fostered by the inclusion of practices and content that are not part of canonical science but were closely related to the activity. For example, in a trip to a forest, the students first heard about the history of the area and the Native peoples' relationships with the forest before European contacts and how that changed over the course of US-Indian history. Students had "relative plants" which they were asked to "visit", while other students collected specimens from other plants, prepared traditional medicines, etc.

In this work the manifestation of the tension related to epistemic structure and standards as well as its resolution were highly contextual. The activity took place in informal educational settings, where content coverage was not an issue; hence, the curriculum developers could choose the science topics to be discussed. A central feature that made the reconstruction of the biology curriculum possible was the specific content in question (i.e., the ecosystem of the forest), a topic central to the community's heritage. This particular topic lent itself to a focus on ecosystems rather than taxonomy and models from a disciplinary perspective. In addition, the life experiences of elders in the community was highly pertinent.

Nevertheless, in our view, even in this case the epistemological tension between personal relevance and disciplinary authenticity remained. Bang and Medin conducted pre and post interviews with the students that revealed a considerable epistemological change. Take for example the following excerpt from a post interview with Sarah, a sixth-grade Choctaw student who grew up in Chicago. Whereas in the interview before the intervention Sarah described science as what is written in the textbooks, and her teacher as the main source of information, her post interview provided a very different picture (Bang \& Medin, 2010, p. 1022):

1. Interviewer: How do you learn about science?

2. Sarah: $\quad$ By my elders and my mom and teachers.

3. Interviewer: What sorts of things do they teach you?

4. Sarah: They teach me about how a long time ago my ancestors how they used to like plant and if there's weeds how they would get it out. They burn ... when plants use to take over they would burn all of them down in one spot.

Sarah words clearly reflect a change towards personal relevance. From a disciplinary authenticity perspective, however, the picture is more complex. While Sarah did make some progress in the sense that she no longer saw science as a school-based activity that is detached from the world, her description seems more in line with practicing history and gardening rather than science. According to our definition of 
disciplinary authenticity, her friend Rachel's ( $7^{\text {th }}$ grade student) epistemological orientation, as expressed in her post interview, reflected a higher sense of disciplinary authenticity, since she describes characteristic aspects of scientific observation (Bang \& Medin, 2010, p. 1022):

Rachel: You can learn about science by just looking around and seeing what is happening. Watching ants grow or working actually watching it - that would take months, but.

In advanced science courses there are topics in which the connections to various cultural resources are less obvious although their appropriation is a prerequisite for any advanced engagement in the discipline. For instance, in the case of physics, astronomy could be a wonderful starting point, but without the formalism of classical mechanics, electromagnetism etc., the students would not be able to professionally engage in physics and engineering. Hence when thinking how to implement this approach in formal science education as a way to enhance personal relevance, immediate tensions related to content coverage arise both from a common school science perspective as well as disciplinary authenticity perspective.

Note also that while there were differences between indigenous and scientific cultures in the ways of knowing and discursive norms related to the topic in question, there was no dispute about the factual description of events, hence content fidelity was not an issue. However, culturally-based knowledge can conflict with scientific canonical knowledge. An example is the challenge of teaching evolution to students from religious families that strongly believe in creationism. The resolution of the tension in these cases can be extremely challenging.

\section{Significance}

A different facet of the tension was discussed by Calabrese-Barton $(1998,2001)$. She provides a critical ethnographic account of a teaching experience in which she and a group of graduate students taught after-school science to children $\left(4^{\text {th }}\right.$ grade) in a long-term homeless shelter. The children's interviews about their experiences in their science lessons illustrate a massive lack of personal relevance that affected their learning considerably (Calabrese-Barton, 2001, p. 901):

Maria and Claudia: We really do hate science! It's boring. All we do is read. Learn about plants and spaceships. It's boring and stupid. We like doing projects but not the cameras [pin-hole camera]. Our boxes were ugly, and all our teacher wanted us to do was to make it just like she said.

In an attempt to create an explicit connection between the activities in the science classroom and the children's daily lives, Calabrese-Barton decided to teach the children about migration, habitat, and local Texas ecosystems through a project in which the children cared for caterpillars. Pets were not allowed in the shelter, and thus apart from providing a context for learning science, caring for the caterpillars responded to the children's desire to have a pet. Calabrese-Barton describes how the children decided to design and build above-ground planters before their caterpillars metamorphosed into butterflies so that they could continue to keep them. One of the children, Claudia, asked if she could use some of the wood and other supplies to build a desk instead, since she did not have a space where she could do her homework. Calabrese-Barton agreed, and the design and building of the desk became Claudia's science project. Calabrese-Barton reports that Claudia learned about scale, measurement, spatial relations, and design, and created an artifact that improved her life from this experience. Moreover, when her family left the shelter they took the desk with them.

The above example illustrates another facet of the tension that we term significance. This facet of the tension reflects a negotiation between the need to make the content and the nature of the activities significant from a school science perspective, while being personally relevant and significant to the students. In other words, we want the students to perceive their activities as contributing, empowering and transformative to their lives. Here, disciplinary authenticity adds another layer of complexity since the activity should be considered legitimate and important also in the discipline. 
The manifestation and resolution of this dilemma are, again, highly contextual. In the case of Calabrese-Barton's work; namely homeless children, after school science, $4^{\text {th }}$ grade students, there is no question that allowing Claudia to build the desk was a correct and powerful pedagogical decision that made the experience relevant at a personal level. However, one can question the extent to what the design and building of the desk were scientifically significant to the topic learned (migration, habitat, and local Texas ecosystems; i.e., common school science perspective), as well as the extent to which this experience engaged Claudia in authentic scientific practice (disciplinary authenticity perspective). In a different context, if Claudia were 17 years old and enrolled in an advanced level biology or physics course, the curriculum could not have emerged solely from her needs and interests (i.e., content coverage), and the scientific significance of the project might have played a much more central role. We are not saying that the question of personal significance is less important in these contexts, but rather that the dilemma and its resolution would have looked very different.

\section{Moving forward}

In the literature reviewed above, the tensions between personal relevance, disciplinary authenticity, and common school science was examined either in the contexts of informal education or at the basic school levels of science and mathematics in traditional American classrooms. We identified several facets of these tensions and argued that their manifestation is highly contextual in terms of the scientific discipline being learned, the scientific level and complexity, the specific attributes of the educational approach (e.g., inquiry), the social infrastructure in which learning takes place, and the students' characteristics. Below, we examine the manifestation of these tensions in two very different examples of instruction and learning of physics at the advanced high school level, in two different countries. The main feature of these examples is the conscious attempt on the part of the teachers to foster the students' sense of personal relevance while maintaining high accountability to the discipline. We believe that a careful examination of the manifestation of the tensions in this context (advanced level STEM education) can problematize their conceptualization and thus contribute to their articulation. It can also enhance our understanding of how to resolve the tensions within the discipline.

\section{The manifestations of the tensions in advanced-level high school physics}

This section discusses two examples of studies that examined the formal learning of physics at the advanced high school level. At first glance, the two cases may seem very different. One focused on redesigning the practice of engaging students in long-term open-ended inquiry projects in physics taking place within the school. The other focused on a teaching experiment that monitored the redesign of a curriculum unit. The first example deals with the practice of inquiry in various topics in physics without any commitment to a particular topic, whereas the second example discusses restructuring the instruction of a particular topic in physics (thermodynamics). Despite these differences, we felt that these two examples have a great deal in common at a foundational level in terms of the instructional goals, the nature of student engagement, the high commitment to disciplinary practices and norms, the focus on developing students' identities, and the concomitant educational research agenda. In particular, in both cases disciplinary authenticity was explicitly considered to be a way to foster and leverage personal relevance in school.

The present work emerged from our attempts to articulate this feeling and decipher the nature of the shared features. Here we aim to uncover the different manifestations of the multifaceted tensions that result from the attempt to establish disciplinary authenticity and personal relevance in each learning environment. As in the literature review, we focus on the facets of the tensions from the teacher's perspective. However, we first need to familiarize the reader with the nature of the specific manifestations of disciplinary authenticity and personal relevance in each example. Both interventions were followed by a study that examined students' engagement and student-teacher interaction. Our data to illustrate the unique and different manifestations of disciplinary authenticity and personal relevance are drawn from these studies, and thus mainly rely on students' and teachers' discourse. In the following sections, we use concrete examples to illustrate how disciplinary authenticity and personal relevance were perceived and experienced 
by the students and teachers in each case, and only then discuss the manifestations of the different facets of the tensions and their resolution in each environment.

\section{Example 1 - Authentic research as an integral part of K12 physics - Reconstructing practice Background}

The first example is drawn from a year-long ethnographic study (Kapon, 2016) that took place in schools associated with the Acheret Center (Acheret Center, 2006). Acheret (the Hebrew acronym for Multi-Cultural Researchers Fellowship) is a regional Israeli center for physics research at the high school level. The center is located in a school. It aims to foster authentic inquiry in advanced $11^{\text {th }}$ and $12^{\text {th }}$ physics classes that take place in the school rather than in outreach centers in universities or in professional research centers. The project advisors are physics teachers rather than professional researchers. As part of their advanced physics studies in high school, all the $11^{\text {th }}$ and $12^{\text {th }}$ grade students in the associated schools conduct an individual or paired long-term research project (18 months). Acheret is organized and functions as a community of learners at the advisor and student level. The leading team believes that mentoring students' research projects is also a venue for the professional development of the mentoring teachers. The study (Kapon, 2016) focused on the learning experiences of students who were mentored by Mr. Alon Schapiro, a physics teacher and an experienced project-advisor in the community who also mentors other project advisors.

Data were collected over a period of one year. The focal participants were the advisor and some of his former $(\mathrm{N}=5)$ and current $(\mathrm{N}=3)$ mentees. The analysis included a content analysis of the final research reports (30-40 pages each), stimulated recall interviews with the former mentees about these reports, participating observation in the weekly meetings at which the current mentees worked with the advisor on their project, in-depth interviews at the end of the year and numerous informal conversations with the advisor after each weekly meeting. Additional data on the social infrastructure in which the advisor and students functioned were collected through informal discussions throughout the year with students of other advisors, discussions with different advisors and the center management team, observation of the students' mock and real oral exams on the projects, and official documents about the center.

The original study was designed using a grounded theory approach (Glaser \& Strauss, 1967) and aimed at answering the following research questions: (1) What did the students learn in this intervention? (2) How did the students perceive the nature of the inquiry they were engaged in and their role in this inquiry? (3) What were the particular features of the mentorship the students received? (4) What were the features of the social infrastructure in which the mentorship and learning took place? We do not attempt to provide a full summary of the findings here since they were published elsewhere (Kapon, 2016). Instead, we will use these data to illustrate the nature of disciplinary authenticity and personal relevance that were nurtured in this learning environment, and then highlight the specific manifestations of the tensions in this environment.

Note that the conceptual framework that guided the original study took a situative interactional approach. The original conceptual framework drew on the closely related ideas of learning in apprenticeship and as part of a community (Lave \& Wenger, 1991; Wegner, 1998), learning in the zone of proximal development (Vygotsky, 1978), and a system perspective on creativity (Csikszentmihalyi, 1997). The categories that emerged from the grounded theory analysis highlighted particular forms of performance and engagement.

\section{The joint manifestation of disciplinary authenticity and personal relevance}

The following subsection provides examples of the students' perceived learning experiences that reflect both personal relevance and disciplinary authenticity. The students' projects dealt with different topics in physics. The analysis also concerned the content of the inquiry, and specifically classified student discourse that reflected understanding or difficulty with (1) the conceptual features, (2) the mathematical modeling, and (3) the experimental procedures of their project. Consider for example an excerpt from a stimulated recall interview with a student in which the student discusses a diagram in his final research report: 
"Then there's this amazing process of the jump to zero degrees [refers to the point on a graph in his report that describes measurements of freezing after super-cooling when the temperature rises abruptly from $-7^{\circ} \mathrm{C}$ to $0^{\circ} \mathrm{C}$. Once it finds a nucleation site, as soon as that happens, the nucleation starts. The freezing process releases a lot of heat energy. It [the temperature] jumps abruptly to zero and stays at zero until all the water freezes. And then if we continue to measure a little longer, it [the temperature] starts to drop down again to // it is ice, but at [a temperature] below zero degrees." (Sam, 328)

Sam's (pseudonym) explanation not only reflects conceptual understanding. His use of the representation (XY graph) to support his explanation and his correct casual use of scientific jargon (e.g., nucleation site) reflects authentic disciplinary discourse. However, what is more interesting is how Sam expressed his explanation, particularly his use of the adjective "amazing" which was repeated throughout the interview whenever the phenomenon of super-cooling came up. The researcher also coded a group of categories that represented discourse that reflected interest, passion, and agency with regard to science. One of these categories was excitement with the actual activity, when the students discussed their scientific work as source of personal enjoyment and satisfaction. Sam's use of the adjective "amazing" with regard to super-cooling is an example of this experience. Note the interaction between disciplinary authenticity and personal relevance that are expressed in the excerpt above. Sam's understanding was not merely technical; the scientific ideas became meaningful to him at a personal level as well, and he expressed his deep connection to the work in different ways throughout the interview. For example (notice the use of "amazing"):

"/.../ working with Alon ((the advisor)), it was amazing, like, it really opens your mind, it's looking at physics as this really cool thing." (Sam, 1112)

A different group of categories in the analysis reflected evidence for the internalization of scientific habits of mind. Consider for example a category labelled students' use of scientific standards of evaluation. Segments classified here included instances in which the students discussed how they employed scientific standards and norms to evaluate the quality of their explanations, claims and measurements. Employing scientific standards for evaluation reflects competence in generating valid scientific knowledge, and thus provides strong evidence for disciplinary authenticity. The following example is drawn from an interview with a student about a project she did on insect aviation:

504. Rina: And, and then I found its 1.98 [a theoretically-based calculation of the drag coefficient she found on the internet]

505. Int. Did it bother you? [her measured drag coefficient was 1.36]

506. Rina: Yes. It bothered me that it, really, ah...

507. Int.: [Laughs] yes...

508. Rina: Like, it's far and...

509. Int.: Yes...

510. Rina: So then I thought /.../ I went back to the video [the videos she took of the movement of a dragonfly], to examine the movement again. Like, I always worked with these videos $/ . . . /$ and I found out. So I went back to the website and saw that they calculated the drag coefficient when it is perpendicular [to the direction of the wind]. And when I looked at the video I saw that it was not exactly perpendicular. So I said, oh, there is something different here. /.../ I went back to the video and the website. Each time I tried to find out what was different between, like "find the differences" and then.. 
511. Int.: So you actually say, that this is something that you initiated, to explain that.

516. Rina: /.../ Like, I, me, I was saying that you cannot just put an item of data in the report without, without...

517. Int.: Without explaining it.

518. Rina: Without comparing it to something that exists. Without explaining it.

Rina (pseudonym) discusses here how she voluntarily used scientific standards of evaluation to regulate and evaluate her progress and the validity of her calculations and measures as she created new knowledge. This excerpt is an example of evidence for the development of sensitivity to the epistemological aspects of reasoning; namely, what counts as "good" evidence in science (i.e., epistemological ideals; see Chinn, Rinehart, \& Buckland, 2014), and a refinement of understanding through model-measurement iterations (i.e., epistemological processes; see Chinn et al., 2014), thus reflecting strong disciplinary authenticity.

However, notice how Rina expressed her thoughts. She was personally invested in this pursuit (e.g., "it bothered me" 506, 508, "like me, I was saying that you can't..." 516). Her concern was not school-like motivation for getting the right answer. To begin with, there were no end of book "right answers". In fact, Rina was the one who initiated the search for another measure that could be compared to her measurement. Rina clearly discusses the norms of reporting a result $(516,518)$. She initiated the search because coming up with a numerical result without estimating its accuracy is not an acceptable scientific practice. Note also how she casually described the process of model-measurement iterative refinement in turn 510. Rina manifested her use of scientific habits of thought that were meaningful to her at a personal level. Other categories in this classification reflect the use of scientific standards of evaluation, refinement of understanding through model-measurement iterations, persistence, sense of esthetics, and abstraction. Most of these utterances were often expressed in a personal idiosyncratic manner that also reflect deep personal relevance.

A different group of categories covered how the students perceived their contribution to the inquiry. Many students described how they took an active part in forming hypotheses and estimates, manufacturing the experimental apparatus, generating the mathematical models, and making decisions regarding the research plan (see for example Rina's turn 510). They also proudly described their contributions to the creative aspects of the project. Such discourse reflects not only the development of creative competence within the discipline - a clear attribute of disciplinary authenticity, but also the development of agency with regard to science and an identity as someone who can do science - an attribute of personal relevance ("Like, I always worked with these videos /.../ and I found out", Rina, 510). This is also apparent in Sam's words: "all this business with electricity etc., this is me" (Sam, 844). Sam was expressing a particular positioning regarding the content that reflected high level agency and deep personal engagement with the content. Strikingly, deep meaningfulness at a personal level was also expressed by students whose conceptual and epistemological development was less impressive.

The interactional approach to the study in which not only the students but also the interactions with the advisor and the supporting social infrastructure were examined provided evidence for the ways in which the engagement in question was fostered, and highlighted the specific positioning of the student relative to the teacher. For example, all the students wrote their reports in the plural. This was not accidental, as the following excerpt reveals:

"The entire report is deliberately written in the plural ... this is a completely joint project.

/.../ I had a partner [refers to the advisor] in every respect." (Rina, 108, 110)

Additional discourse markers (categories) highlighted that the students perceived the project as an authentic inquiry and the advisor as a collaborator in this inquiry. These discourse markers included the 
deliberate use of the plural, explicit acknowledgement of the advisor's unique contribution, and descriptions of instances of joint thinking. The latter discourse marker is particularly interesting since sometimes the students positioned the advisor relative to themselves as the knowledgeable guide: "He always tried to get me, like, to try to think, and to see the way to go, and do it alone" (Rina 140). At other times they positioned the advisor as a peer investigator: "Alon [the advisor] was the one who was like writing the equations, but this was like brainstorming" (Gur, 576). This relative positioning was also mirrored in the advisor's discourse.

Comparing the advisor's framing of the interaction to that of the students revealed a very high level of intersubjectivity (Wertsch, 1984). Like the students, the advisor considered the project to be an authentic joint inquiry and thought of the projects as his own as well (again, a particular positioning). Hence, the fact that the teacher-advisor was learning with the students seemed to foster a sense of authentic inquiry and relevance.

The advisor's discourse when meeting with the students constantly indicated to them that what they were doing had disciplinary meaning and relevance outside the classroom as well. For instance, when a pair of students told the advisor that they might need to do an experiment similar to one conducted by another pair, he told them they could cite their peers in their report, similar to their citations of other scientific papers, and some of them did this. He encouraged a student to send an email with a question to a university physics professor with particular expertise. Moreover, when he and other advisors in the community talked about the projects (with the students, as well as with peers), they referred to the students' projects as research studies, and explicitly used the Hebrew word for academic research. Students presented their work in progress, consulted with their peers, other advisors and experts in the community, and were tested on their final product by the community members as well as by external evaluators. Hence, the community of teacher-advisors filled the role of experts in professional scientific work (Csikszentmihalyi, 1991). These norms and discourse generated a personal, social, deeply affective, and future-oriented engagement with the scientific content for the students.

\section{The manifestation of the tensions}

Although the original analysis (Kapon, 2016) was not explicitly designed to examine the issue of tensions in the original study, a reexamination of the data uncovered specific manifestations of all the facets of the tensions discussed in the literature review.

\section{Content fidelity}

One line of tension emerged between cultivating students' interest and agency with regard to science (e.g., positioning the students as generators of knowledge) and remaining accountable to the discipline. This tension often manifested itself in terms of content fidelity. For instance, the focus advisor placed more emphasis on how the students felt about the project and whether it was an original creation of their own than on whether the project would make an original or important contribution to the field of physics. Hence, at times he preferred personal relevance over disciplinary authenticity. He also openly acknowledged that occasionally mistakes in physics could be made even in the final report, due to the complexity of some of the topics the students chose to study. Hence the tension with respect to content fidelity was between personal relevance (how the students felt about the project and what they learned from it) and traditional schooling that privileges "correct answers." Like many of the other advisors, he resolved this tension by arguing that the skills the students learn along the way, and their developing confidence in their abilities are at least as important as acquiring an accurate conceptual understanding of the topic.

The skills and habits of mind that the advisor was alluding to are an essential part of the discipline. Recall for example how Rina (the student who studied the aviation of insects) described the back-and-forth movement between the theoretical model and the empirical results, how her interpretation of both changed during this process, and how she constantly searched for other independent comparable measurements. In the same spirit Sam described how he was constantly engaged in critical assessment of the validity of his observations: 
Disciplinary authenticity and personal relevance

There are quite a few small experiments that we did that do not appear in this report. We did them just to see, to prove things to ourselves, to be absolutely sure that this is how it ((the phenomenon)) takes place" (Sam, 1096)

Doing authentic scientific work from the advisors' and from the students' perspective involved not only generating new ideas but also the conscious and systematic evaluation of these ideas. This evaluation was explicitly also facilitated by the design of the social infrastructure in which the students and their project advisor worked. There were several points during the work on the projects in which the students and/or their teacher-advisor had to present their ideas, concerns and findings to other students, project-advisors, expert guests, and external evaluators. These people collegially but openly critiqued the work, highlighting pitfalls and suggesting possible alternatives and solutions. This process is quite close to how peer review functions in science, either formally (as in journals and talks), and informally (as in research groups and informal consultations with peers.) Hence the tension of content fidelity was resolved within the discipline by acknowledging that making mistakes is an inevitable part of doing science, but also by acknowledging that science offers tools and cultural ways to regulate the process of generating new ideas and evaluating their validity, and by acknowledging that students should explicitly engage in this process.

\section{Content coverage}

A different manifestation of the tension relates to content coverage. Working on the projects requires time; thus, Acheret students did not study $30 \%$ of the topics covered by their peers in traditional advanced level physics classrooms. The conflict here was between disciplinary authenticity, which requires in-depth time-consuming learning, and common school science which requires a certain coverage. Additionally, since the projects were tailored to suit particular students' abilities and backgrounds, they differed in depth and complexity. Hence, there was also a tension between personal relevance that is sensitive to students' abilities and interests, and common school science that privileges standardized assessments. Acheret (partially) resolved this tension through negotiations with the Israel Ministry of Education that resulted in a local accommodation of the external requirements for matriculation in advanced level physics: the students were required to take the external matriculation exams in mechanics and electricity and magnetism (60\%), and their grade on the project (report and oral exam) replaced the external matriculation exams in radiation and matter and laboratory skills (40\%).

\section{Language and discursive norms}

As described above, the discourse between the students and the advisor pointed to a social framework of joint authentic inquiry. The report the students submitted for external evaluation was a product of shared work with the advisor, and the students wrote it as such. This creates a conflict with traditional external assessment since it was almost impossible to differentiate between the advisor's and the students' contributions to the final product. The traditional approach to assessment may thus question the validity of the students' final grade as a reflection of the students' skills and conceptual understanding.

This example illustrates a manifestation of tensions related to language and discursive norms. First, it is a tension between language and discursive norms in authentic scientific practice (i.e., disciplinary authenticity) and common school science. Disciplinary authenticity privileges collaborative work including cases in which one of the authors is a knowledgeable apprentice (e.g. a graduate student) and the other is an expert (i.e., a professional scientist). Common school science on the other hand privileges individual external assessment as the main tool for evaluating students' progress. Second, it is a tension between personal relevance and common school science. In principle, the students could write their reports in the singular, and use phrasing that described their work solely as their own. In fact, some of them said in the interview that they were explicitly advised to do so by the teacher in the school in charge of the formal submission of the projects for external evaluation. Their refusal and insistence that "this is a joint project" (Rina, above) reflects their integrity and the personal attachment to the project. 
Disciplinary authenticity and personal relevance

\section{Epistemic structure and standards}

The examination of the students' reports also reveals a manifestation of the epistemic structure and standards facet of the tension. From the perspective of disciplinary authenticity, references to scientific work should be textbooks, or peer reviewed scientific journals. However, references such as Wikipedia, You Tube, etc. are appreciated much more from the perspective of personal relevance since these are what teenagers use all the time. The advisors in Acheret resolved this tension by acknowledging both as legitimate resources for information, and by admitting that they use these resources as well. The initial references that the students examined were often Wikipedia, You Tube, etc.. But as the work progressed, the need for more professional and focused references naturally emerged and each final report cited at least one "serious" scientific paper that the students read carefully.

\section{Significance}

Many advisors mentioned the challenge of formulating authentic research problems that stemmed from the interests expressed by the students but were still within the capacity of high school physics students. This challenge illustrates the significance facet of these tensions, particularly between disciplinary authenticity and personal relevance. Many advisors (partially) resolved this tension by acknowledging that the problems the students addressed were not authentic in the sense that they did not reflect what professional physicists are currently studying but were authentic in terms of the methods used to solve the problems and the fact that the solution was unknown. The latter point exemplifies the depth of the contextuality of the tension. In the context of physics classroom teaching, even at the undergraduate levels, no one expects that problems students solve should reflect what professional physicists are currently studying. However, in the context of authentic research apprenticeships, this is not the case. Most research apprenticeships offered to excellent high school students in outreach programs at research universities invite outstanding secondary school and undergraduate students to join an authentic research group and take an actual part in an ongoing project. This was not the case in Acheret, where the research projects were done in school and were crafted for this purpose. Whereas studies that have examined the participation of secondary school and undergraduate students in research apprenticeships in authentic research groups characterized the students' involvement as peripheral legitimate participation, where students dealt mainly with the technical side of the work (Sadler, Burgin, McKinney, \& Ponjuan, 2010), the students here took active part in formulating the research questions, making decisions regarding the next steps of the research, etc.

Some advisors encouraged their students to reexamine problems that engaged physicists during the $17^{\text {th }}-19^{\text {th }}$ centuries but by using modern tools unavailable to physicists at that time (e.g., computerized sensors, high-speed video cameras, etc.). This approach is attuned to students' capacities but is less sensitive to their personal interests. The focus advisor (Alon) took a different approach. He began with problems that interested the students and worked with them to articulate a quantitative research question and design an experiment that could answer this question (often based on a quick internet search). Based on these very rough measurements, he and the students discussed what was interesting about the results, what they were curious about investigating further, and how they could improve their measurements, in a process that closely reflect authentic scientific problematizing (Phillips, Watkins, \& Hammer, 2017). During this process, the specific theme of the project usually emerged as the student's own idea. For example, Steve ( $11^{\text {th }}$ grade) began by wanting to build a suit that would allow him to breathe freely underwater. He ended up studying factors that influence the efficiency of water electrolysis. This approach cultivated students' agency and ownership of the projects considerably. Nevertheless, it was quite time-consuming. Moreover, about half of the projects ended in a dead end due to the students', and sometimes also the advisor's limited knowledge or limited access to equipment, thus necessitating a change of topic in the middle of the year, which challenged the common school science perspective. 


\section{Example 2 - Authentic learning in thermodynamics - Reconstructing curriculum Background}

The second example is taken from a project conducted by the Physics Education Research Group at the University of Bologna. In 2008, the group carried out a teaching experiment based on extensive development of a curricular unit on thermodynamics. The teaching experiment was carried out in an authentic classroom of 20 students (17 year olds) at a scientifically oriented secondary school in Rimini, Italy, and the implementation took about 25 hours. The teacher was involved in both designing the unit and analyzing the data (Fantini, 2014).

The disciplinary content was restructured to address two overarching goals. The first was to create a rich learning environment that could foster a deep conceptual understanding of thermodynamics by problematizing and comparing the macroscopic and microscopic approaches. This was accomplished by using multiple contexts and definitions for exploring the same concept in a variety of circumstances and by drawing on diverse resources such as applets, original texts by physicists such as Boltzmann and Maxwell, and critical essays on the distinction between the microscopic and macroscopic approaches to thermodynamics.

The second goal was to promote and nurture equity by making the environment psychologically safe and inclusive (Nasir, Rosebery, Ann, Warren, \& Lee, 2006). The research group adopted the perspective of learning as a cultural process (Nasir et al., 2006). Thus, they pursued equity by making the epistemological structure visible and by challenging the authoritative and exclusive image of science in which only one point of view is legitimate (and possible). "Voices" of eminent scientists such as Maxwell, Boltzmann, Clausius, Kelvin and Einstein became part of classroom discussions by analyzing excerpts from these scientists' original papers on thermodynamics and comparing their different interpretations of thermodynamic phenomena.

During the implementation, several types of data were collected: written assignments on conceptual problems and exercises, written questionnaires about the epistemological differences between the microscopic and the macroscopic approach to thermodynamics, audio and video recording of all the lessons, notes taken by the researcher who observed all the lessons and eight final semi-structured individual interviews.

As in the first example, this study was originally designed according to the principles of the grounded theory approach (Glaser \& Strauss, 1967). The analysis (Levrini et al., 2015) aimed to explain a phenomenon that impressed the researchers during the intervention. They noticed that in addition to demonstrating a thorough understanding of basic concepts in thermodynamics, the students appeared to make sense of the learning materials in a personal way and used idiosyncratic words and sentences in their speech instead of the "school" language that students generally use by borrowing expressions from the teacher or the textbooks. The notion of appropriation elaborated by Bakhtin (1981) was adapted and operationalized to account for this phenomenon, which seemed to enlarge conceptual learning to incorporate personal authenticity and relevance. ${ }^{1}$ According to Bakhtin:

"It [a word] becomes 'one's own' only when the speaker populates it with his own intentions, his own accent, when he appropriates the word, adapting it to his own semantic and expressive intention. Prior to this moment of appropriation, the word [...] exists in other people's mouths, in other people's contexts, serving other people's intentions: it is from there that one must take the word, and make it one's own" (Bakhtin, 1981, p.293-94).

\footnotetext{
${ }^{1}$ Note that the words "appropriation" and "authenticity" share the same etymological meaning: the Latin "proper" and the Ancient Greek "autòs" have the same meaning "of one's own."
} 
This definition of appropriation contrasts with the more typical connotation of the appropriation of scientific discourse, which implies that students learn to speak like scientists or use words in the formal register of physics without necessarily developing personal meaning. However, according to Bakhtin, appropriation implies that accountability is situated in the students and reveals a special sense of belonging and active participation: "I make a [scientific] word mine."

Appropriation was operationally conceptualized and defined by Levrini et al. (2015) through an analytic process of bootstrapping five discourse markers from interviews and excerpts of classroom discussions. These markers capture how students' language is both disciplinarily authentic and personally relevant in the sense that they show how the curriculum and its thoughtful implementation effectively foster the development of disciplinary knowledge and epistemological competences, thus supporting the development of students' identity (Levrini et al., 2018).

\section{The joint manifestation of disciplinary authenticity and personal relevance}

Appropriation can be seen, in retrospect, as the manifestation of a special type of engagement that combines disciplinary authenticity and personal relevance. The combination of the two was evident in the final individual interviews, when students were asked to reflect on their learning path and describe the meanings they arrived at for defining basic thermodynamic concepts such as temperature.

Almost all the students interviewed (6 out of 8) discussed the concept of temperature in a way that was valid with respect to the discipline but was not a repetition of a textbook or teacher definition of the concept. In their answers, the students selected precise pieces of knowledge and coordinated them so as to infuse a disciplinary word with personal preferences and goals. This was also evident in students who did not have outstanding results or particularly good grades in physics (Levrini et al., 2015). Levrini et al. coded personal relevance by identifying frequent words or expressions in students' discourse which, when associated, highlighted a personal authentic positioning with respect to physics (thermodynamics). More specifically, positioning was considered personally authentic and relevant when the use of words and expressions was unique to the specific student, reflecting linguistic choices that could not have been borrowed from an external authority (classmate, teacher, parent, textbook...). For example, Matteo's (pseudonym) discourse was filled with words like philosophy, speculation, reflections, reasoning, being and becoming, which did not characterize either the teacher's discourse or that of other students. When discussing the concept of temperature, Matteo correctly compared two laws he had learned in which temperature appears: the ideal gas law $(\mathrm{PV}=\mathrm{nRT})$ and the law of calorimetry $(\mathrm{Q}=\mathrm{mc} \Delta \mathrm{T})$. This comparison allowed him to stress the dual property of temperature as a quantity that describes an equilibrium state or as a cause for a process. In the distinction between state and process, Matteo recognized a possible place to identify his personally relevant connection to the philosophical notions of becoming (change) and being (state). Matteo saw the law of calorimetry $(\mathrm{Q}=\mathrm{mc} \Delta \mathrm{T})$ as an expression of becoming:

"There is a change [because of $\Delta \mathrm{T}$ ] that means everything is not stable and everything is not being, there is something that changes" (Matteo).

In contrast, in thinking about the ideal gas law $(\mathrm{PV}=\mathrm{nRT})$, Matteo saw an expression of being:

"[There is] absolute temperature T, that doesn't change. There is no $\Delta$ [difference in temperature], there is no change ..." (Matteo).

To emphasize what we mean by personal relevance and idiosyncrasy, let us consider the discourse of another student, Michele (pseudonym). He used words like objects, engines, work, etc. frequently in his discourse. His interest in engines and real objects strongly influenced his engagement with the content. When discussing what he had learned about temperature, he focused on the role of temperature in making thermal engines work:

"Different temperatures are necessary /.../ only with different bodies with different temperatures can we have a cycle of work; different temperatures induce a heat exchange - as we call it - and the heat exchange induces work; heat is turned into work" (Michele).

The students who appropriated thermodynamics like Matteo and Michele not only employed an internal persuasive discourse that revealed deep conceptual understanding but seemed to be aware of the intellectual choice they had made and demonstrated epistemological consciousness. This consciousness 
was traced by coding students' utterances that expressed specific positioning with respect to physics in general (and not only thermodynamics), that involved the relationship between theory and experiments, the role of models and theories in organizing knowledge and supporting consistency, the relationship between knowledge and reality, the place in science for different interpretations of the same formalism, and the scope of science and its relationship to applications. In monitoring epistemological consciousness, the researchers paid special attention to whether and how the epistemological utterances were consistent with students' personal ways of engaging with the content. The results were interesting since the epistemological claims were consistently used by the students to legitimize individual ways of learning and attaching personal meanings to physics contents. The researchers inferred that the epistemological dimension allowed the students to combine personal relevance and disciplinary authenticity, since it opened up new interpretative spaces for exploring personal issues without subverting scientific constraints. To illustrate and exemplify this, we reconsider the cases of Matteo and Michele.

When discussing ideas that were coded as relating to the epistemology of physics, Matteo emphasized the role of philosophical speculations in science:

"It [the approach to thermodynamics they experienced in class] was nice since it leads people to reflect on things that, in some areas [like physics], you do not think of. A physics made up of formulas is different from a physics made up of reasons, of [arguments that show] how one arrives at these formulas" (Matteo).

In this quotation, Matteo moves from thermodynamics to physics in general and expresses his personal engagement ("it was nice") as a form of emotional and cognitive resonance with one facet of physics by emphasizing the philosophical considerations that underlie the formulas rather than the formulas per se. For Matteo, dealing with authentic scientific debates became a way to develop an epistemological view of physics that legitimized his personal authentic and relevant engagement with the contents.

Michele, in the final interview, often repeated sentences like these:

"I like physics because it explains how reality works. That is to say, I'm very curious about

how objects work and about natural events...."

Michele's statement shows a specific epistemological stance that refers to physics in general (and not only on thermodynamics) ("I like physics") and to the scientific habits of mind that he searched for and preferred in the discipline ("it explains how reality works"). Michele likes to discover how things, phenomena and engines work and the mechanisms that regulate them. The relationship between technology and science, as well as the focus on reality and the experimental aspects of science made his experience in disciplinary learning more personally appealing.

The students' personal engagement with conceptual and epistemological ideas was not accidental. The teacher carefully orchestrated moments in the discussion in which students were invited and guided to verbalize, analyze, and defend their own position (Levrini, Levin, Fantini, \& Tasquier, under review). These moments were fundamental in nurturing students' agency and their awareness of their own personal position with respect to physics. For example, the teacher stimulated and supported a debate between Matteo and Michele. During this discussion, the students did not only discuss pure scientific ideas. They also expressed themselves, their preferences, and their dreams for the future as they discussed the scientific ideas.

As we will argue more extensively in the following section, the curriculum materials were fundamental to supporting personal engagement, particularly the multi-perspective lens provided by comparing different approaches, and the multi-dimensionality provided by the use of different resources concerning different dimensions in physics (e.g., conceptual, formal, experimental, applicative, and historical-epistemological resources).

It is worth noting that in this educational context, the meaning of authenticity emerged from the interaction between canonical science, the teacher and the students. Authenticity took place in a rich learning environment in which students took ownership of what they learned, and expressed it in their own voice (Buxton, 2006; Calabrese-Barton, 2001; Rivera Maulucci et al., 2014). However, this intervention did not take place in the context of informal education, but rather in a typical school setting, within an 
authentic classroom, in which students coped with the hierarchical structure of a challenging discipline like physics.

\section{The manifestation of the tensions}

Levrini et al. (2015) did not examine the issue of tensions. In fact, they only realized its existence in retrospect when taking the entire design and implementation of the intervention into consideration, since many of the facets of these tensions were resolved at the level of the design or through the vast experience of the teacher who allowed these tensions to be systematically dealt with. In this section we examine the facets of the tensions that became evident through this retrospective analysis. The teacher was involved in controlling the reliability of the analysis and providing feedback throughout this study.

\section{Content fidelity}

This facet of tension was addressed in the class through the establishment of classroom norms that allowed content fidelity to be experienced as the collective process of pointing out the "rules of the game". In an $a$-posteriori interview, the teacher used an unusual metaphor to describe how she aligned content fidelity with a search for personal meaning that could also lead the students to an elaboration of what was rigorously disciplinary "correct": "tirare e mollare la corda" ("pulling the reins tight and letting them go slack") (Levrini et al., under review). Some classroom moments were explicitly devoted to sharing a sense of disciplinary constraints by summing up the learned disciplinary contents ("pulling the reins"). At those times, the students were required to take part in the construction of a collective narrative that recapped the scientific contents and gave back a sense of content fidelity. At other times, the students were encouraged to play freely with the ideas ("letting the reins go slack") by exploring possible interpretations and by following "unofficial" and unexpected routes. These moments were fostered mainly through the discussions of epistemological texts by different authors, for example the criteria distinguishing the microscopic and macroscopic approaches to thermodynamics. Two classroom norms were adhered to strictly by both the teacher and the students:

1. Suspension of the teacher's judgment so that the students could trust the teacher's message of letting them play freely with their ideas;

2. Distributed awareness that each student could and was expected to make a contribution to the collective discussion and dynamics of the classroom.

These kinds of norms were deliberately established by the teacher, as she stated during the interview:

"When students are trying to play with their ideas, it is obvious that I have to suspend my judgment. I tell them that it is a difficult game but that it is worthwhile for them, for their growth. So I have to respect it... I have to be consistent with the message I want to convey... In this sense, every kind of judgment must be suspended. It is not a question of assessing performance, but rather helping them to grow. My idea is that they have to assume the responsibility for that. They do not always have to seek my approval. If they feel comfortable with their stories, the stories are ok. I only have to help them respect the constraints posed by physics. This is not easy, but I do believe this is our role as teachers."

At the beginning of every discussion during which the students were encouraged to play with their ideas, the teacher stressed very clearly that she was not going to evaluate what they would say specifically, but their level of involvement, the coherence of their thinking and the contribution they were able to make to the collective discussions. Right-wrong ideas were not at issue, but rather the ability to consistently argue a position. Only after a position was made explicit, was its consistency with the constraints imposed by a robust scientific argumentation verified. This process was stressed as authentically scientific, since the free exploration of ideas in science acts to generate new knowledge, and in order to be accepted, any new piece 
of knowledge needs to be analyzed to test its inner logical consistency, its coherence with the established body of knowledge, and its significance and robustness in terms of reality.

\section{Content coverage}

This facet of the tension played an important and explicit role during the design phase. The tension related to content coverage between common school science and disciplinary authenticity was resolved by the designers' decision to respect the institutional constraints. The thermodynamics unit took about 25 hours (about 3 months and a half), a reasonable time within the Italian mandatory syllabus when the experiment took place $(2008)^{2}$. The official Italian curriculum is fairly flexible and not completely pre-determined. It defines general learning outcomes concerning skills and only stipulates a minimal list of topics that have to be covered. As a result, teachers have some latitude to decide on the level of detail, emphasis, and instructional methods they employ.

To avoid or circumscribe tension related to content coverage, the research team restructured the thermodynamics curriculum in close collaboration with the teacher, with the explicit goal of covering the official content (Levrini, Fantini, Pecori, \& Tasquier, 2014). They decided not to exceed the degrees of freedom that the official syllabus permits for epistemological activities, which were designed to enhance the students' personal engagement. Furthermore, the curriculum also intentionally included familiar and accepted forms of evaluation such as textbook exercises and problems. Hence, the content coverage facet of the tension was present in the design phase of the intervention, and due to its resolution during this phase was no longer an issue in the implementation phase.

\section{Epistemic structure and standards}

The most challenging tension presented by the restructured curriculum on thermodynamics had to do with regard to epistemic structure and standards and was manifested in different ways both at the design and the instructional practice levels.

The first issue that caused tension was the image of the discipline presented in the restructured curriculum. The image of physics that is widely accepted in common school science, and supported by most textbooks depicts physics as univocal, non-historical, and as a collection of facts and laws of nature that are accepted as true because of their empirical grounding or mathematically based derivation. By contrast the image of physics advocated by the restructured curriculum is that of a dialogic, historical and argumentative discipline, whose empirical and mathematical groundings play a central argumentative role: they are only part, although a fundamental and essential one, of the scientific discourse and what science is about. This image coheres better with a disciplinary authenticity perspective.

Two design principles in the teaching materials explicitly challenged the conventional textbook image of physics. The first was multi-perspectiveness. This design principle refers to the choice of presenting the macroscopic and the microscopic approaches to thermodynamics as two different perspectives from which the same problems are addressed. The second design principle was multidimensionality. This principle refers to the choice of analyzing the two perspectives not only for their conceptual contents but also for their different underlying epistemological structure as regards their different explanatory models, the different relationships between theoretical hypotheses and empirical data, and so on. The univocal image of physics was challenged during classroom activities that explicitly considered the different voices of Einstein, Maxwell, Boltzmann, Clausius and Kelvin, and their personal interpretations of the problems in question. The students' engagement with these different perspectives was facilitated through specific activities. For example, an epistemological questionnaire that required the

${ }^{2}$ A recent school reform has altered the syllabus. The major innovation has to do with the official introduction of $20^{\text {th }}$ century physics (special relativity, quantum physics and elements of nuclear physics) in the final year (grade 13). This has placed tighter constraints on the total number of hours a teacher can devote to a topic and the tension related to content coverage has increased considerably in the last few years. However, the remaining freedom in the choice of the specifics, approaches and methods still allows the official contents to be covered without having to abandon the design principles consistent with the aim of fostering personal engagement (Levrini, Malgieri et al., in preparation). 
students to analyze and compare original papers in which these scientists argued about the difference between the microscopic and macroscopic approaches formed the core of individual and collective discussions.

Note that the epistemic tension between these two images of physics requires resolution on the part of the teacher as well (De Ambrosis \& Levrini, 2010), since the required epistemological knowledge and skills are not part of the conventional program of teacher education. Since the teacher was part of the design team from the very beginning, her epistemic basis of the curriculum was aligned with that of the group. However, this epistemic tension remains highly challenging for other teachers who wish to use the curriculum.

Another manifestation of the epistemic structure and standards tension was manifested during the teacher's interaction with specific students. Here the perspective of personal relevance added additional complexity. For example, Matteo's approach to knowledge often reflected a tendency toward speculative philosophical investigations, which led him at times to try to evade the technical - experimental or formal - aspects of physics. This personal tendency created tensions between personal relevance and school science, as well as between personal relevance and disciplinary authenticity. In common school science, written exercises and problems are the main form of evaluation, and these require the use of mathematical formalisms and laboratory work. The disciplinary authenticity perspective privileges arguments that are grounded in mathematical formalism and experimental evidence. Matteo would have preferred to ignore the mathematical and experimental aspects of physics, and to remain mainly in the speculative philosophical regime of the argument. Hence, the teacher had to convince him that formal scientific arguments are important and powerful. The fact that the mathematical and experimental aspects of physics were a central part of the assessment was not enough to convince Matteo to engage deeply with them. Faced with this dilemma, the teacher opted for a special way to interact with Matteo during her dialogical lectures and to keep him at close rein with the discipline, which , a- posteriori, was recognized by the researchers as a form of revoicing (O'Connor \& Michaels, 1996), which was deliberatively strategized by the teacher to guide Matteo to ground his claims in mathematical derivations and experimental evidence (Levrini, Levin, Fantini, \& Tasquier, under review). For example, consider the following excerpt taken from a dialogical interactive lecture in which the teacher and Matteo discuss the efficiency of an ideal thermal engine:

1. Matteo: The efficiency in a cycle cannot be greater than 1, but (pause). However, this is also in an ideal cycle.

2. Teacher: Good. It cannot be. (pause) We are considering an ideal cycle, an ideal, reversible cycle as Carnot did. So?

3. Matteo: It [the efficiency] must necessarily be smaller than 1

4. Teacher: Exactly. Smaller than 1, not higher and not even equal.

5. Matteo: Exactly, not even equal

Matteo (turn 1) started by a making a slightly incorrect claim. The teacher (turn 2) immediately recognized the imprecision (the efficiency has to be smaller than 1, it cannot be equal to 1). She reacted by revoicing Matteo ("Good. It cannot be.") in order to support and encourage him. However, after that, she invited him (turn 2, second half) to rephrase his answer, by calling his attention to a particular aspect of the problem in question (i.e. "we are considering an ideal cycle, an ideal, reversible cycle").

Matteo understood his mistake and corrected himself (turn 3). The teacher, in turn 4, again supported and encouraged him by revoicing his last words ("Exactly. Smaller than 1", turn 4), as well as by highlighting the substantial aspects of the idea ("not higher and not even equal", turn 4). Matteo understood this point ( "Exactly, not even equal", turn 5) but their interaction did not end there. The teacher used it to leverage his acknowledgement of the formal basis of thermodynamics as a powerful argumentative tool to control and strengthen his claims: 
6. Teacher: If it were equal [pause]. Tell me, efficiency is equal to// [the teacher takes a piece of chalk and turns to the blackboard, ready to write what Matteo would say]

7. Matteo: Work is equal to work over heat absorbed. [the teacher writes on the blackboard $\eta=$ $\left.\frac{W}{Q_{a b s}}\right]$

8. Teacher: Good. Work is equal to [pause]. Explain in terms of heat.

9. Matteo: Heat absorbed minus heat lost [the teacher goes on to write on the blackboard: $\eta=$ $\left.\frac{W}{Q_{a b s}}=\frac{Q_{a b s}-Q_{\text {lost }}}{Q_{a b s}}<1\right]$

10. Teacher: If the efficiency were 1 , which of these terms would be zero?

11. Matteo: The heat lost [pause]. And this is not possible.

In turns 6-9 the teacher invited Matteo to define efficiency mathematically: she writes on the blackboard what Matteo dictates to her. In turn 10 she explicitly asks him to use the mathematical representation to refute his original claim (turn 1), and Matteo successfully does so.

Turns 5-10 exemplify how the teacher resolved the tension between personal relevance and disciplinary authenticity as regards the epistemic facet. The teacher built on Matteo's interest in speculation and gradually guided him to recognize the formal structure of a scientific argument while acknowledging the power of scientific argumentation as a special way to refute or support a speculative claim. By resolving this epistemic tension between pure speculative claims and scientific arguments, the teacher helped this student who had already manifested his personal interest in philosophy to find his own place within physics.

\section{Language and discursive norms}

The dialogical nature of the teaching materials fostered the creation of a special language with literature references, key words and linguistic contexts that do not belong to the language and discursive norms of traditional physics textbooks or, more generally the traditional school culture. This poses a potential conflict when students are evaluated by external teachers. In Italy, this may become an issue at the end of secondary school, where part of the final graduation exam in physics includes an oral colloquium which is evaluated by an external teacher. The teacher tried to resolve this in two ways. The first was to make students conscious of the linguistic norms and discourse that characterize typical textbooks. Students were guided to read, discuss and comment on examples, as well as to compare textbook language and discourse with other materials such as original historical memoirs or epistemological texts. The second way the teacher used to reduce possible sources of tension was to prepare a detailed description of the curriculum for the external evaluators to facilitate the dialogue between them and the students.

\section{Significance}

In general students do not spontaneously consider thermodynamics as directly impacting their lives. Nevertheless, the authentic dialogical view of science enacted by multi-perspectiveness and multidimensionality presented opportunities for the students to situate themselves within the discipline and to activate a process of identification within the disciplinary contents. In particular, the presence of multiple voices from different scientists encouraged students to search for their own personal voice and legitimized their discussions of physics as genuine thinkers. As shown in the case of Matteo with regard to the epistemic facet of the tension, sometimes the search for personal significance (i.e., Matteo's preference for a particular kind of argument) could conflict with the disciplinary habits of mind and scientific argumentation that are highly significant to authentic scientific work. This tension between disciplinary constraints and the freedom to search for personal relevance and self-expression in the content was resolved by the teacher by exploiting what she considered to be the essence of scientific argumentation: expressing a personal thesis 
in scientific terms adhering to the formal and experimental constraints that make science a special form of knowledge.

A different manifestation of the significance facet of tension came from students who liked and felt comfortable in traditional common school science or had a strong objective image of science where interpretations and debates do not play a structural role. For these students, a univocal presentation of science represents an efficient way to get the right answer or the essence of a phenomenon quickly. These students may perceive the multi-dimensionality and multi-perspectiveness of the curriculum to be a waste of time, for example, when preparing for a future scientific career. This was a serious tension that the teacher had to resolve for some students on different temporal scales from individual lesson to the time scale of the entire intervention.

On the time scale of individual lessons, the teacher had to capture the mood of the class and, accordingly, manage the pace for example by changing the linguistic register or the focal dimension of the lesson if the tension became too great. Throughout the entire intervention, the teacher made sure to explicate and convince the students that epistemic, argumentative and dialogical skills are important and contribute to problem solving and the development of a deeper understanding of scientific ideas at the technical level as well. During the lessons she often stressed the epistemological and argumentative competence of seminal scientists (Maxwell, Einstein, ...), when the class analyzed these scientists' original writings. One of the things that facilitated the resolution of this tension was that the students and their parents were aware of the teacher's solid reputation and recognized success in preparing students for university tests and exams. A less well-known teacher might have experienced greater resistance.

\section{Discussion}

The goal of this paper was to problematize the conceptualizations the tensions between disciplinary authenticity, personal relevance, and common school science. Both the literature we reviewed and our own studies highlight the multifaceted and contextual nature of these tensions. We identified five facets of these tensions: content fidelity, content coverage, language and discursive norms, epistemic structure and standards, and significance. The different ways each facet is realized in the perspectives of disciplinary authenticity, personal relevance, and common school science (summarized in Table 1) create multidimensional tensions that are not easy to resolve. 
Table 1. Facets of the tensions between personal relevance, disciplinary authenticity, and common school science.

\begin{tabular}{|c|c|c|c|}
\hline Facet & Personal relevance & Disciplinary authenticity & $\begin{array}{c}\text { Common school } \\
\text { science }\end{array}$ \\
\hline $\begin{array}{l}\text { Content } \\
\text { fidelity }\end{array}$ & $\begin{array}{l}\text { Students should } \\
\text { develop their agency } \\
\text { as individual thinkers } \\
\text { who can create } \\
\text { knowledge. The } \\
\text { question of the fidelity } \\
\text { of the knowledge } \\
\text { created is irrelevant. }\end{array}$ & $\begin{array}{l}\text { Students should learn the content } \\
\text { and practices of science. Content } \\
\text { fidelity either: (1) Adheres with } \\
\text { canonical, culturally agreed } \\
\text { upon knowledge. (2) Emerges } \\
\text { from the authentic empirical and } \\
\text { dialectical practices that guide } \\
\text { and inform the creation, } \\
\text { elaboration, refinement and } \\
\text { evaluations of new ideas. Hence, } \\
\text { errors and inaccuracies are an } \\
\text { inherent part of this dynamic } \\
\text { process, though authentic } \\
\text { measures are taken to avoid } \\
\text { them. }\end{array}$ & $\begin{array}{l}\text { Students should learn } \\
\text { correct and acceptable } \\
\text { scientific ideas as they } \\
\text { are expressed in the } \\
\text { school curriculum. }\end{array}$ \\
\hline $\begin{array}{l}\text { Content } \\
\text { coverage }\end{array}$ & $\begin{array}{l}\text { The curriculum is } \\
\text { largely emergent and } \\
\text { discovered "on the } \\
\text { fly" based on students' } \\
\text { ideas, interests and } \\
\text { difficulties. }\end{array}$ & $\begin{array}{l}\text { The curriculum is flexible and } \\
\text { largely emerges from students' } \\
\text { discoveries, authentic } \\
\text { explorations and/or authentic } \\
\text { sense-making activities. }\end{array}$ & $\begin{array}{l}\text { The curriculum is } \\
\text { fully predetermined, } \\
\text { reflecting a demand } \\
\text { for a specific } \\
\text { coverage and } \\
\text { emphases. }\end{array}$ \\
\hline $\begin{array}{l}\text { Language } \\
\text { and } \\
\text { discursive } \\
\text { norms }\end{array}$ & $\begin{array}{l}\text { The accepted language } \\
\text { and discursive norms } \\
\text { come from the culture } \\
\text { to which the students } \\
\text { belong. }\end{array}$ & $\begin{array}{l}\text { The accepted language and } \\
\text { discursive norms represent the } \\
\text { richness and scope of the culture } \\
\text { of the scientific discipline. }\end{array}$ & $\begin{array}{l}\text { The accepted } \\
\text { language and } \\
\text { discursive norms are } \\
\text { the ones that } \\
\text { characterize common } \\
\text { school science. }\end{array}$ \\
\hline $\begin{array}{l}\text { Epistemic } \\
\text { structure and } \\
\text { standards }\end{array}$ & $\begin{array}{l}\text { The accepted cultural } \\
\text { ways of experience } \\
\text { and knowing are those } \\
\text { that characterize the } \\
\text { culture to which the } \\
\text { students belong, } \\
\text { and/or identify with. }\end{array}$ & $\begin{array}{l}\text { The accepted cultural ways of } \\
\text { experience and knowing are } \\
\text { those that characterize the } \\
\text { culture of the scientific } \\
\text { discipline. }\end{array}$ & $\begin{array}{l}\text { The accepted cultural } \\
\text { ways of experience } \\
\text { and knowing are those } \\
\text { that characterize } \\
\text { common school } \\
\text { science. }\end{array}$ \\
\hline
\end{tabular}




\begin{tabular}{|llll|}
\hline Facet & Personal relevance & \multicolumn{1}{c|}{$\begin{array}{c}\text { Disciplinary authenticity } \\
\text { Significance }\end{array}$} & $\begin{array}{l}\text { Common school } \\
\text { science }\end{array}$ \\
$\begin{array}{l}\text { The content and the } \\
\text { nature of the activities } \\
\text { the students engage in } \\
\text { students as } \\
\text { contributing, } \\
\text { empowering and } \\
\text { transformative to their } \\
\text { lives }\end{array}$ & $\begin{array}{l}\text { The content and the nature of the } \\
\text { activities the students engage in } \\
\text { can be seen as central or } \\
\text { contributing to the content and } \\
\text { practice of science. }\end{array}$ & $\begin{array}{l}\text { The content and the } \\
\text { nature of the activities } \\
\text { the students engage in } \\
\text { are specified in the } \\
\text { curriculum and are } \\
\text { internally and } \\
\text { externally assessed. }\end{array}$ \\
\hline
\end{tabular}

We begin this discussion by a reexamination of the notion of disciplinary authenticity and what it entails for science education. In the introduction we stated that aiming for disciplinary authenticity in education in our view means designing and facilitating learning experiences that immerse students not only in the conceptual and epistemological features of science, but also in its social dialectical practices, as well as its affective features. We discussed Debora Ball's (1993) and David Hammer's (1997) work on discovery learning and used it to highlight the tension related to content fidelity and content coverage. Hammer (2001) discussed the tension between scientific practices and traditional content-oriented concerns. We interpreted this tension as a tension between personal relevance and common school science, rather than as a tension inherent to disciplinary authenticity. Our examples illustrated how disciplinary authenticity can offer ways to resolve tensions related to content fidelity by acknowledging that the practice of doing science involves not only the generation of new ideas but also their conscious evaluation, and the translation of this recognition into the design and practice of teaching science.

Mihaly Csikszentmihalyi's (1997) socio-constructivist account of creativity suggests that creativity in a cultural domain (in our case, physics) is the product of a complex system of interactions between (1) a domain - a culturally evolved discipline that consists of a set of symbolic rules and procedures; (2) a field - all the individuals who act as the gatekeepers of the domain and decide whether a new idea or product should be included in the domain; and (3) the individual - the person who uses the symbols and procedures of a given domain to express a new idea, or notice a new pattern, which is accepted as novel by the field. Both the Israeli and the Italian examples present a conscious educational implementation of Csikszentmihalyi's notion of field. In the Israeli example the social infrastructure (i.e., the structured feedback from the members of the community and gusts) created the field, and in the Italian example it was the teacher's "pulling the reins tight" after "letting them go slack." The Israeli example also highlighted students' discourse that demonstrated conscious employment of authentic means to regulate empirical work and evaluate theoretical insights.

From a disciplinary authenticity perspective content fidelity emerges from the authentic empirical and dialectical practices that guide and inform the creation, elaboration, refinement and evaluations of new ideas. Errors and inaccuracies are an inherent part of this dynamic process; however, the Israeli and Italian examples suggest that, as in authentic scientific practice, students can meaningfully engage in explicit use of authentic means to systematically minimize these errors. The educational challenge is similar in a way to the scientific one when considering how to balance these processes; namely, deciding when to slacken the reins and when to tighten them.

We feel that scientific disciplines are epistemologically and culturally rich enough to accommodate a significantly wider range of discursive norms and epistemic moves than what is offered by common school science. The Warren et al. (2001) description of Emilio's participation in science exemplifies the 
narrow perception of scientific disciplines in common school science. Although the student's cultural discursive moves cohered with authentic scientific practices (thought experiments), the problem was that these were not considered scientific practices in school science. Bang and Medin (2010) discussed a restructuring of curriculum to enable it to capitalize on indigenous students' cultural ways of knowing; specifically, by using a system-level analyses and ecosystems instead of a taxonomic approach as an organizing principle, exemplifying an epistemic resolution within the discipline.

Note however, that since Bang and Medin's curriculum was designed for informal education, and was an educational context that did not prescribe a particular content coverage, they could choose a scientific topic that was central to their students' heritage (the ecosystem of the forest). The instructional design of the curriculum on thermodynamics that we described in the Italian example (Levrini \& Fantini, 2013) exemplifies that while there are topics that are more obviously connected students' lives and culture, no scientific topic should be considered as precluding a sense of personal relevance. Levrini et al. (2015) showed that when the instruction of any topic in science reflect its breadth, depth, complexity, and dialectical nature, it not only enhances students' knowledge and understanding, but also makes the learning environment inclusive to a larger group of students by helping them to find their own voice within the discipline.

We stress that there is no specific single prescription for the design of learning experiences that foster a sense of disciplinary authenticity and personal relevance which fit all topics and all educational contexts. Different scientific topics offer different affordances for different instructional contexts, and some topics are easier to reconstruct than others for a given context. The point is that examining these topics through the lens of disciplinary authenticity, with a clear yet complex vision of personal relevance, and a deep understanding of the educational context in question can reveal these affordances and be used to later capitalize on them.

The examination of the resolution of the different facets of the tension in the literature and in our own examples stressed the central role of teachers in the successful negotiation between disciplinary authenticity, personal relevance and school science. The kind of teaching described in both examples necessitated an articulated form of expertise from the teachers that reflected not only pedagogical expertise but also deep and broad disciplinary understanding. The teachers in both examples were required to move through and between several dimensions of physics, such as its historical-philosophical grounding and its experimental and applied dimensions. This flexibility requires a very strong foundation of content knowledge (though not all- encompassing), and a deep, broad and flexible understanding of the practices and epistemology of the discipline. The teachers were also sensitive to, interested in, and responded to the students' thinking. They presented strong orchestration skills that allowed them to navigate between all these aspects of the instruction in real time (Levrini, Levin, Fantini, \& Tasquier, under review).

Our work also highlights that while disciplinary authenticity often offers pathways to resolve the tension between school science and personal relevance, it requires thoughtful negotiation since it may create a tension with personal relevance. Consider for example the case of Matteo, the student in the Italian example, who found the mathematical formalism and empirical work tedious and tended to avoid it altogether. The teacher resolved this tension through her interaction with Matteo. She creatively and thoughtfully negotiated and guided Matteo to productively use and acknowledge the power of scientific argumentation as a special and productive way to investigate his own reasoning. Another example of negotiation between personal relevance and disciplinary authenticity concerned the issue of legitimate resources of information in the case of research apprenticeships in school in Israel. By acknowledging Wikipedia, You Tube, and other informally accepted sources of information as legitimate initial sources of information for the students' research work, the advisors had time to establish, as the work on the research progressed, a real need for more professional and focused references. These considerations are particularly illuminating when considering the question of relevance from a youth-centered perspective (a term that was suggested by Buxton, 2006). Limiting the discussion of science solely to what immediately interests and feels relevant to students may suspend the challenge of engaging students in science rather than confronting it. What our examples highlight is the importance of cultivating a sense of relevance and meaning. 
Throughout the paper we argued that the manifestations of the tensions are highly contextual; thus, their resolution may differ in different contexts of instruction. Our reading of the literature suggests that these tensions have mainly been investigated in informal education or in elementary and basic secondary levels in American classrooms. We, on the other hand, examined their manifestation in educational contexts that involve the instruction of science (physics) at the advanced high school levels, and in different countries. Our examination problematizes and enriches the debate about the conceptualization of these tensions because it uncovers aspects that are less apparent in educational contexts in which the tensions were previously studied, due to the stringent need for accountability to the discipline that teaching physics at this level requires.

Although the Israeli and Italian examples concerned the instruction of physics at the advanced high school level, the facets of the tensions were manifested in very different forms in each, as a result of the different contextual constraints of each intervention. Consider for example the tension related to epistemological structure and standards. The main tension in the Italian classroom were manifested with regard to the image of science. Students who liked and felt comfortable in traditional school science or preferred a strong objective and univocal image of science resisted the reformed curriculum at first, and the teacher had to negotiate the instructional approach and convince them that engaging in the dialectic aspects of science was a productive activity. Note that the need to negotiate the reformed curriculum to the "natives of common school science" can be seen as an inverse mirror to the tension Bang and Medin (2010) described in their study. The centrality of the epistemic feature of the tension in the Italian case is not surprising given that the most innovative aspects of the design were the application of multi-perspectiveness and multi-dimensionality of the physics content. In the Israeli intervention the tension was manifested around decisions about valid sources of knowledge in physics, and how the distribution of the work between the advisor and the students could be translated into assessment of individual students. This is not surprising either, considering that the essence of the intervention was "doing science through research" where the students were generating knowledge, and that authentic science privileges collaborative work while common school science mainly assesses individuals' achievements.

Context also influenced the students' interpretations of what counts as an important activity, to whom, and according to whom (i.e., the significance facet of the tension). Consider for example CalabreseBarton's (2001) study discussed in the literature review. The contextual features there were young children, living in a shelter for homeless families, participating in an after- school science club. Claudia and Maria, the young girls in the study, perceived their science teacher's behavior as judging and uncaring, and thus felt that her science lessons were irrelevant to their lives ("boring and stupid.") The contextual features of the Italian case were almost the opposite since they were advanced level physics students, oriented towards learning in the university, who had involved parents. Here the manifestation of the significance tension presented an inverse image. Students who liked and felt comfortable in traditional common school science felt at times that the multi-dimensionality and multi-perspectiveness of the new curriculum was a waste of time, for example when preparing for a future scientific career (i.e., external exams). The teacher in the Italian case, unlike the teacher in Calabrese-Barton's study, had a very strong emotional connection with the students and they and their families trusted and respected her. The tension was successfully resolved since this trust gave her the time she needed for the thoughtful negotiation she conducted between the two perspectives. This points to the pivotal role of the teacher in resolving (or not resolving) the tension, and the extent to which the manifestation of the significance tension was different in these very different contexts.

Another example of the influence of the context on the perception of significance has to do with whether an educational activity is significant from the perspective of disciplinary authenticity. Physics presents a hierarchical structure of knowledge in which advanced ideas are built on the foundations of more basic ideas. Hence in most cases at the high school and introductory undergraduate level students do not (and cannot) work on state-of-the-art scientific problems, whereas other aspects of disciplinary authenticity could be dealt within these settings. However, in research apprenticeships for high school students that take place in the university and professional outreach programs, students' projects often address the state-ofthe-art questions. This is possible since the participating students are mainly involved in the technical sides 
of the research (Feldman et al., 2013; Sadler et al., 2010). In the Israeli case the research apprenticeships took place in school and were crafted specifically for this purpose. The advisors were highly aware of this expectation and openly admitted that the studies they mentored were far from the state-of-art in terms of the focus of the study. However, they used the school context to resolve this tension, and emphasized that the methods of investigation were highly authentic, and that their students were able to gradually participate in all phases of the research, in ways that participating in a state-of-the-art study would not have afforded.

While the Israeli and Italian cases, in which we examined the tensions in detail, concerned the instruction of physics at the advanced secondary school level, they were very different in terms of the focus and goals of the instruction. Nevertheless, both aimed to promote the students' conceptual and epistemological development and the development of creative competences within the discipline while at the same time nurturing the development of the students' personal development as individuals, citizens and future professionals. The Israeli case exemplifies how high school students can engage in authentic research, and how this experience can become a transformative experience at both the disciplinary and personal levels. The Italian case exemplifies how the teaching of a predefined topic that is central to the discipline and is not explicitly related to students' lives can still become a venue in which students explore and develop their identities.

These cases of teaching physics at the advanced high school level highlight that personal significance can be nurtured during the process of learning rather than considered as an a-priori condition. Science teaching and learning are traditionally oriented toward canonical content rather than to aspects of "doing science" or "developing a personal view on science". In our view, this is not an either/or question. Developing students' conceptual and epistemological understanding is crucial since without this understanding we cannot teach science. In fact, this is our main concern with regard to the youth-centered approach to authenticity (Buxton, 2006). Focusing on the voice of the students should in no way diminish focusing on the discipline. Indeed, this is exactly the tension we wish to resolve. The question is how students' voices can be heard within the discipline and what forms of participation in the discipline will allow them to develop their authority and autonomy. Without developing students' disciplinary-based creative competences we deprive them of understanding the essence of doing science. On the other hand, without nurturing students' personal, social and professional identities and sense of agency, science will not become meaningful to them and will not affect how they imagine their future.

\section{Acknowledgments}

We deeply thank David Hammer for his thoughtful comments on previous versions of this manuscript. An earlier version containing some of the ideas in this manuscript was presented in a short paper at the 2016 International Conference of the Learning Sciences in Singapore (Kapon, Laherto, \& Levrini, 2016). Olivia Levrini wishes to thank Paola Fantini, the teacher of the thermodynamics class, for her careful reading, feedback and verification of the accuracy of the analysis. Shulamit Kapon wishes to thank Alon Schapiro, the talented and inspiring project advisor, and his students who warmly and openly shared their thoughts and experiences with her, as well as Moshe Reich and Dr. Amos Cohn for inviting her to study the unique learning experience in physics that they nurture at the Acheret center. The preparation of this paper was supported by the Israel Science Foundation under grant \#325/17.

\section{References}

Acheret Center. (2006). Retrieved October 20, 2016, from http://www.acheret.org.il/

Allchin, D. (2011). Evaluating knowledge of the nature of (whole) science. Science Education, 95(3), 518-542. https://doi.org/10.1002/sce.20432

Bakhtin, M. (1981). Discourse in the novel. In M. Holquist (Ed.), The dialogic imagination (pp. 259422). Austin, TX: University of Texas Press.

Ball, D. L. (1993). With an Eye on the Mathematical Horizon: Dilemmas of Teaching Elementary School Mathematics. The Elementary School Journal, 93(4), 373-397. https://doi.org/10.1086/461730

Ball, D. L., Sleep, L., Boerst, T. A., \& Bass, H. (2009). Combining the Development of Practice and the 
Practice of Development in Teacher Education. The Elementary School Journal, 109(5), 458-474. https://doi.org/10.1086/596996

Bang, M., \& Medin, D. (2010). Cultural processes in science education: Supporting the navigation of multiple epistemologies. Science Education, 94(6), 1008-1026. https://doi.org/10.1002/sce.20392

Bang, M., Medin, D., \& Washinawatok, K. (2010). Innovations in culturally based science education through partnerships and community. In M. S. Khine \& I. M. Saleh (Eds.), New science of learning: Cognition, computers and collaboration in education (pp. 569-592). New York: Springer. Retrieved from http://link.springer.com/chapter/10.1007/978-1-4419-5716-0_28

Barab, S. A., \& Hay, K. E. (2001). Doing science at the elbows of experts: Issues related to the science apprenticeship camp. Journal of Research in Science Teaching, 38(1), 70-102.

Bell, R. L., Blair, L. M., Crawford, B. A., \& Lederman, N. G. (2003). Just do it? Impact of a science apprenticeship program on high school students' understandings of the nature of science and scientific inquiry. Journal of Research in Science Teaching, 40(5), 487-509.

Bleicher, R. E. (1996). High school students learning science in university research laboratories. Journal of Research in Science Teaching, 33(10), 1115-1133.

Brown, J. S., Collins, A., \& Duguid, P. (1989). Situated cognition and the culture of learning. Educational Researcher, 18(1), 32-42.

Buxton, C. A. (2006). Creating contextually authentic science in a "low-performing" urban elementary school. Journal of Research in Science Teaching, 43(7), 695-721.

Buzzoni, M. (2015). Causality, Teleology, and Thought Experiments in Biology. Journal for General Philosophy of Science, 46(2), 279-299. https://doi.org/10.1007/s10838-015-9293-9

Calabrese-Barton, A. (1998). Teaching science with homeless children: Pedagogy, representation, and identity. Journal of Research in Science Teaching, 35(4), 379-394.

Calabrese-Barton, A. (2001). Science education in urban settings: Seeking new ways of praxis through critical ethnography. Journal of Research in Science Teaching, 38(8), 899-917.

Charney, J., Hmelo-Silver, C. E., Sofer, W., Neigeborn, L., Coletta, S., \& Nemeroff, M. (2007). Cognitive apprenticeship in science through immersion in laboratory practices. International Journal of Science Education, 29(2), 195-213.

Chinn, C. A., \& Malhotra, B. A. (2002). Epistemologically authentic inquiry in schools: A theoretical framework for evaluating inquiry tasks. Science Education, 86(2), 175-218.

Chinn, C. A., Rinehart, R. W., \& Buckland, L. A. (2014). Epistemic cognition and evaluating information: Applying the AIR model of epistemic cognition. In D. Rapp \& J. Braasch (Eds.), Processing inaccurate information (pp. 425-454). Cambridge, MA: MIT Press.

Crawford, B. A. (2012). Moving the essence of inquiry into the classroom: Engaging teachers and students in authentic science. In K. C. D. Tan \& M. Kim (Eds.), Issues and challenges in science education research (pp. 25-42). Dordrecht: Springer.

Csikszentmihalyi, M. (1991). Flow: The psychology of optimal experience. New York, NY: Harper Perennial.

Csikszentmihalyi, M. (1997). Creativity: Flow and the Psychology of Discovery and Invention. New York, NY: Harper Perennial.

De Ambrosis, A., \& Levrini, O. (2010). How physics teachers approach innovation: An empirical study for reconstructing the appropriation path in the case of special relativity. Physical Review Special Topics - Physics Education Research, 6, 020107. Retrieved from https://journals.aps.org/prper/abstract/10.1103/PhysRevSTPER.6.020107

Duschl, R., \& Grandy, R. (2011). Demarcation in science education: Toward an enhanced view of scientific method. In R. Taylor \& M. Ferrari (Eds.), Epistemology and science education: Understanding the evolution vs. intelligent design controversy (pp. 3-19). New York: Routledge.

Edelson, D. C. (1998). Realizing authentic science learning through the adaptation of scientific practice. In B. J. Fraser \& K. G. Tobin (Eds.), International handbook of science education (pp. 317-331). Dordrecht, NL: Kluwer.

Erduran, S., \& Dagher, Z. R. (2014). Reconceptualizing the nature of science for science education: 
Scientific knowledge, practices and other family categories. Dordrecht, NL: Springer.

Fantini, P. (2014). Verso una teoria locale dell'appropriazione nell'insegnamento/apprendimento della fisica [Toward a local theory of appropriation in the teaching/learning of physics]. Bergamo, Italy: University of Bergamo.

Feldman, A., Divoll, K. A., \& Rogan-Klyve, A. (2013). Becoming researchers: The participation of undergraduate and graduate students in scientific research groups. Science Education, 97(2), 218243.

Gilbert, J. K., \& Reiner, M. (2000). Thought experiments in science education: potential and current realization. International Journal of Science Education, 22(3), 265-283. https://doi.org/10.1080/095006900289877

Glaser, B. G., \& Strauss, A. L. (1967). The discovery of grounded theory: Strategies for qualitative research. New York: Aldine de Gruyter.

Hammer, D. (1997). Discovery Learning and Discovery Teaching. Cognition and Instruction, 15(4), 485529. https://doi.org/10.1207/s1532690xci1504

Hammer, D. (2001). Student Inquiry in a Physics Class Discussion. Cognition and Instruction, 13(3), 401-430.

Hammer, D., \& Zee, E. van. (2006). Seeing the science in children's thinking : case studies of student inquiry in physical science. Portsmouth, NH: Heinemann. Retrieved from http://www.heinemann.com/products/e00948.aspx

Hunter, A., Laursen, S. L., \& Seymour, E. (2007). Becoming a scientist: The role of undergraduate research in students' cognitive, personal, and professional development. Science Education, 91(1), $36-74$.

Hurd, P. D. (1998). Linking science education to the workplace. Journal of Science Education and Technology, 7(4), 329-335.

Irzik, G., \& Nola, R. (2014). New directions for nature of science research. In M. Matthews (Ed.), International handbook of research in history, philosophy and science teaching (pp. 999-1021). Dordrecht, NL: Springer.

Jaber, L. Z., \& Hammer, D. (2016). Learning to Feel Like a Scientist. Science Education, 100(2), 189220.

Kapon, S. (2016). Doing research in school: Physics inquiry in the zone of proximal development. Journal of Research in Science Teaching, 53(8), 1172-1197. https://doi.org/10.1002/tea.21325

Kapon, S. (2017). Unpacking Sensemaking. Science Education, 101(1), 165-198. https://doi.org/10.1002/sce.21248

Kapon, S., \& diSessa, A. A. (2012). Reasoning Through Instructional Analogies. Cognition and Instruction, 30(3), 261-310.

Kapon, S., Laherto, A., \& Levrini, O. (2016). Conceptualizing Authenticity and Relevance of Science Education in Interactional Terms. In C.-K. Looi, J. Polman, U. Cress, \& P. Reinmann (Eds.), The International Conference of the Learning Sciences (ICLS) (pp. 843-846). Singapore: International Society of the Learning Sciences.

Lave, J. (1997). The culture of acquisition and the practice of understanding. In D. Kirshner \& J. A. Whitson (Eds.), Situated cognition: Social, semiotic, and psychological (pp. 17-36). Mahwah, NJ: Lawrence Erlbaum Associates.

Lave, J., \& Wenger, E. (1991). Situated learning: Legitimate peripheral participation. Cambridge, UK: Cambridge university press.

Lee, H.-S., \& Butler, N. (2003). Making authentic science accessible to students. International Journal of Science Education, 25(8), 923-948. https://doi.org/10.1080/09500690305023

Levrini, O., \& Fantini, P. (2013). Encountering productive forms of complexity in learning modern physics. Science \& Education, 22(8), 1895-1910.

Levrini, O., Fantini, P., Pecori, B., \& Tasquier, G. (2014). Forms of Productive Complexity as Criteria for Educational Reconstruction: The Design of a Teaching Proposal on Thermodynamics. Procedia Social and Behavioral Sciences, 116, 1483-1490. https://doi.org/10.1016/J.SBSPRO.2014.01.421 
Levrini, O., Fantini, P., Pecori, B., Tasquier, G., \& Levin, M. (2015). Defining and Operationalizing 'Appropriation' for Science Learning. Journal of the Learning Sciences, 24(1), 93-136.

Levrini, O., Levin, M., \& Fantini, P. (2018). Personal, deeply affective, and aesthetic engagement with science content: when disciplinary learning becomes a vehicle for identity construction. In T. G. Amin (Ed.), Converging Perspectives on Conceptual Change. Mapping an Emerging Paradigm in the Learning Sciences (p. in press). London: Routlege.

Levrini, O., Levin, M., Fantini, P., \& Tasquier, G. (n.d.). Orchestrating classroom discussions to foster appropriation. Under Review.

Mann, C. R. (1914). What is industrial science? Science, 515-524.

McAllister, J. W. (1996). The evidential significance of thought experiment in science. Studies in History and Philosophy of Science Part A, 27(2), 233-250. https://doi.org/10.1016/0039-3681(95)00044-5

Meltzer, D. E., \& Otero, V. (2015). A brief history of physics education in the United States. American Journal of Physics, 83(5), 447-458.

Nasir, N. S., Rosebery, Ann, S., Warren, B., \& Lee, C. D. (2006). Learning as a cultural process: Achieving equity through diversity. In R. K. Sawyer (Ed.), The Cambridge handbook of the learning sciences (pp. 489-504). Cambridge: Cambridge University Press.

NGSS Lead States. (2013). Next Generation Science Standards: For States, By States. Washington DC: The National Academies Press.

O'Connor, M. C., \& Michaels, S. (1996). Shifting participant frameworks: Orchestrating thinking practices in group discussion. In D. Hicks (Ed.), Discourse, learning, and schooling (pp. 63-103). New York, NY: Cambridge University Press.

Phillips, A. M., Watkins, J., \& Hammer, D. (2017). Problematizing as a scientific endeavor. Physical Review Physics Education Research, 13(2), 020107. https://doi.org/10.1103/PhysRevPhysEducRes.13.020107

Rahm, J., Miller, H. C., Hartley, L., \& Moore, J. C. (2003). The value of an emergent notion of authenticity: Examples from two student/teacher-scientist partnership programs. Journal of Research in Science Teaching, 40(8), 737-756.

Richards, J., \& Robertson, A. D. (2016). A review of research on responsive teaching in science and mathematics. In A. D. Robertson, R. E. Scherr, \& D. Hammer (Eds.), Responsive Teaching in Science and Mathematics (pp. 36-55). New York: Routlege.

Rivera Maulucci, M. S., Brown, B. A., Grey, S. T., \& Sullivan, S. (2014). Urban middle school students' reflections on authentic science inquiry. Journal of Research in Science Teaching, 51(9), 11191149.

Rosebery, A. S., Warren, B., \& Conant, F. R. (1992). Appropriating Scientific Discourse: Findings From Language Minority Classrooms. Journal of the Learning Sciences, 2(1), 61-94. https://doi.org/10.1207/s15327809j1s0201_2

Sadler, T. D., Burgin, S., McKinney, L., \& Ponjuan, L. (2010). Learning science through research apprenticeships: A critical review of the literature. Journal of Research in Science Teaching, 47(3), 235-256.

Sandoval, W. A., \& Reiser, B. J. (2004). Explanation-driven inquiry: Integrating conceptual and epistemic scaffolds for scientific inquiry. Science Education, 88(3), 345-372.

Stuckey, M., Hofstein, A., Mamlok-Naaman, R., \& Eilks, I. (2013). The meaning of 'relevance'in science education and its implications for the science curriculum. Studies in Science Education, 49(1), 1-34.

Vygotsky, L. S. (1978). Mind in society: The development of higher mental process. Cambridge, MA: Harvard University Press.

Warren, B., Ballenger, C., Ogonowski, M., Rosebery, A. S., \& Hudicourt-Barnes, J. (2001). Rethinking diversity in learning science: The logic of everyday sense-making. Journal of Research in Science Teaching, 38(5), 529-552. https://doi.org/10.1002/tea.1017

Watkins, J., Coffey, J. E., Redish, E. F., \& Cooke, T. J. (2012). Disciplinary authenticity: Enriching the reforms of introductory physics courses for life-science students. Physical Review Special Topics Physics Education Research, 8(1), 010112. https://doi.org/10.1103/PhysRevSTPER.8.010112 
Wegner, E. (1998). Communities of practice: Learning, meaning and identity. Cambridge, UK: Cambridge University Press.

Wertsch, J. V. (1984). The zone of proxiaml development: some conceptual issues. In B. Rogoff \& J. V Wertsch (Eds.), Children's learning in the" zone of proximal development (pp. 7-17). Jossey-Bass Inc Pub. 